June 22, 2021

\title{
Light neutral CP-even Higgs boson within Next-to-Minimal Supersymmetric Standard model (NMSSM) at the Large Hadron electron Collider ( $\mathrm{LHeC}$ )
}

\author{
Siba Prasad Das 11 and Marek Nowakowski12 \\ ${ }^{1}$ Department of Physics, Faculty of Science, \\ Universidad de los Andes, Apartado Aereo 4976-12340, Carrera 1 18A-10, \\ Bogota - Colombia. \\ ${ }^{2}$ M. Smoluchowski Institute of Physics, Jagiellonian University, ul. St. Lojasiewicza 11, \\ 30-348 Kraków - Poland.
}

\begin{abstract}
We analyze the prospects of observing the light CP-even neutral Higgs bosons $\left(h_{1}\right)$ in their decays into $b \bar{b}$ quarks, in the neutral and charged current production processes $e h_{1} q$ and $\nu h_{1} q$ at the upcoming LHeC, with $\sqrt{s} \approx 1.296 \mathrm{TeV}$. Assuming that the intermediate Higgs boson $\left(h_{2}\right)$ is Standard Model (SM)-like, we study the Higgs production within the framework of NMSSM. We consider the constraints from Dark-matter, Sparticle masses, and the Higgs boson data. The signal in our analysis can be classified as three jets, with electron (missing energy)coming from the neutral (charged) current interaction. We demand that the number of b-tagged jets in the central rapidity region be greater or equal to two. The remaining jet is tagged in the forward regions. With this forward jet and two $b$-tagged jets in the central region, we reconstructed three jets invariant masses. Applying some lower limits on these invariant masses turns out to be an essential criterion to enhance the signal-to-background rates, with slightly different sets of kinematical selections in the two different channels. We consider almost all reducible and irreducible SM background processes. We find that the non-SM like Higgs boson, $h_{1}$, would be accessible in some of the NMSSM benchmark points, at approximately $0.4 \sigma(2.5 \sigma)$ level in the $e+3 \mathrm{j}$ channel up to Higgs boson masses of $75 \mathrm{GeV}$ and in the $\mathbb{H}_{T}+3 \mathrm{j}$ channel could be discovered with $1.7 \sigma(2.4 \sigma)$ level up to Higgs boson masses of $88 \mathrm{GeV}$ with $100 \mathrm{fb}^{-1}$ of data in a simple cut-based (with optimization) selection. With ten times more data accumulation at the end of the LHeC run and using optimization, one can have $5 \sigma$ discovery in the electron (missing energy) channel up to 85 (more than 90) GeV.
\end{abstract}

\footnotetext{
${ }^{1}$ Email: sp.das@uniandes.edu.co

${ }^{2}$ Email: mnowakos@uniandes.edu.co
} 


\section{Introduction}

It is expected since long that the mechanism that triggers the electroweak symmetry breaking (EWSB) and generates the fundamental particle masses will involve at least two experimental parts. The first one is the search and the observation of a spin-zero Higgs particle that will confirm the scenario of the minimal SM (which has one Higgs isospin doublet) of GlashowWeinberg-Salam and most of its extensions. This confirms a spontaneous symmetry breaking by a scalar field that develops a non-zero vacuum expectation value (vev). This part has recently been closed by the ATLAS and CMS experiments [1, 2] at Large Hadron Collider (LHC) with the spectacular observation of a new boson with present central mass value around $125.09 \pm 0.21 \pm 0.11 \mathrm{GeV}$. The width, and the couplings to all SM particles and the CP-quantum numbers are also known. All this seems consistent with the symmetry breaking mechanism in the SM and opens up the second part: are there any other scalars from beyond the SM model, which would participate in the symmetry breaking? This second part is mandatory in order to establish the exact nature of the electroweak symmetry breaking (EWSB) mechanism and, eventually, identify the effects of new physics beyond the SM.

The original idea of having scalars in the model is, of course, the spontaneous breaking of the electroweak gauge group. A detailed overview has been given in [3] and in particular the non-standard way of the EWSB in [4]. However, scenarios where the scalar do not participate in the EWSB do exist (see for instance, neutrino models where the vevs of singlet scalars breaks spontaneously the lepton number [5]).

Also worth mentioning are the higher dimensional theories, based on the Standard Model gauge group [6] where the electroweak constraints can be consistent with experimental results, even without Higgs boson. In this kind of models, the electroweak symmetry is broken by the boundary conditions and the choices of compactification scales lead to the masses of the gauge bosons.

The available theoretical models at our disposal are many: generic two Higgs doublet Model (2HDM) [7, 8] and various flavor violating Yukawa-textured models [9], Minimal Supersymmetric Standard Model (MSSM) [10], non-minimal realization of supersymmetric models, and models with additional singlets, the Next-to-Minimal Supersymmetric SM (NMSSM), doublets and/or triplets [11, 12, 13, 14, 15] and non-minimal NMSSM type of models, e.g., in [46], 47]. Hence, one of the most important tasks for experimentalists and theorists is to find ways to either exclude or confirm aspects of these models which may have varieties of signatures for the different Higgs production and decay channels at the present and the upcoming collider experiments.

From the perspective of model building, the NMSSM [11, 12, 13, 14, 15] is ideally suited to search for new physics as its gauge group is the same as in the SM and thus it can easily accommodate the SM-like discovery without any unnatural fine-tuning of its parameters. Although the MSSM contains less free parameters than the NMSSM, the SM Higgs boson type signal can also be easily accommodated in the latter model whereas some amount of fine tuning is necessary for the MSSM. Some variants of the NMSSM models also have nice features of the Higgs sector. Worth mentioning is the model with a slightly broken PQ-symmetry [16] and the so-called $\lambda$-NMSSM [17].

It is worth pointing out that in the NMSSM the upper limit of the lightest SM-like Higgs

boson mass is lifted up to $155 \mathrm{GeV}$ (as compared to $139 \mathrm{GeV}$ in the MSSM). Secondly, the 
problem with the absence of Sparticle signals may easily be explained by different Supersymmetry (SUSY) cascade decays occurring in the NMSSM, owing to an additional singlino entering as the last step and thereby inducing topologies to which present SUSY searches are less sensible. Furthermore, as is well known, the MSSM suffers from the so-called $\mu$-problem, i.e., the Higgs(ino) mass term entering the soft SUSY Lagrangian ought to be manually set at the EW scale in order to achieve EWSB, while SUSY itself would require it to be at the Planck scale (or else be zero, in virtue of some postulable additional symmetry) [18, 19]. This is elegantly remedied in the NMSSM, since herein the aforementioned soft term is replaced by the vev of an additional Higgs singlet state, which appears naturally at the EW scale. In turn, this implies that the Higgs sector of the NMSSM is very rich. In fact, while only one Higgs boson exists in the SM and five Higgs bosons in the MSSM, there are seven such states in the NMSSM: three CP-even Higgses $h_{1,2,3}\left(m_{h_{1}}<m_{h_{2}}<m_{h_{3}}\right)$, two CP-odd Higgses $a_{1,2}\left(m_{a_{1}}<m_{a_{2}}\right)$ and two charged Higgses $h^{ \pm}$.

As the SM is embedded within any two-Higgs doublet model, the recently discovered SMHiggs boson can be part of the spectrum. Generically, this SM-type Higgs is either the lightest CP-even neutral one or the second-lightest one. Light as well as heavy Higgs boson phenomenology within the MSSM has been studied extensively in [20]. Having many free parameters in NMSSM, the masses of the Higgs bosons vary in a wide range so that their decay branching ratios in various modes can also vary widely. The Higgs boson masses together with their couplings to gauge boson and/or fermions are most important to identify the viable modes to look for the Higgs boson in any collider experiment. From the theoretical perspective, the two-loop corrected Higgs boson masses and couplings to quarks and gauge bosons within NMSSM have been carried out in [21, 22.

The NMSSM Higgs boson phenomenology at high energy colliders has been studied over a decade [23, 24, 25, 26, 27, 28, 29, 30] and direct experimental searches are reported in [31].

From the upcoming experiment perspective, the LHeC facility [32] is expected to be operational at CERN around 2020. It will be a Deep Inelastic Scattering (DIS) experiment at the $\mathrm{TeV}$ scale, with the center-of-mass energy of around $1.3 \mathrm{TeV}$. In comparison, another recently closed (in 2007) DIS experiment (the Hadron-Electron Ring Accelerator (HERA) 33] at DESY had a center-of-mass energy of around $320 \mathrm{GeV}$ with an integrated luminosity of around 0.5 $\left.\mathrm{fb}^{-1}\right)$. The LHeC might deliver data samples of approximately $100 \mathrm{fb}^{-1}$ and at the end of full data accumulation with $1000 \mathrm{fb}^{-1}$ (with a higher detector coverage). Taking into considerations all kinematical and detector aspect details, the overall kinematic range (in $x$ and $Q^{2}$ ) at LHeC is 20 times larger than the HERA experiment. Other than the in-depth studies of QCD, the LHeC also has an enormous scope to probe electroweak and Higgs boson physics [34, 35, 36.

One of the nicest features of almost all SUSY models, is that the neutral lightest Sparticle state is naturally the dark matter candidate [37, 38]. Within the standard cosmological scenario, we assumed that the dark matter candidate is the lightest neutralino, $\tilde{\chi}_{1}^{0}$ with the correct abundance of relic density consistent with recent Planck measurement [39]. We refer to [40, 41] where the dark matter phenomenology within NMSSM has been studied. Some variant models are discussed in [42] and a discussion on low mass weakly interacting massive particle (WIMP) searches consistent with the Higgs boson data at LHC has been studied recently in [43]. Sparticle co-annihilation consistent with the dark matter relic-density and related SUSY phenomenology in the electroweak gaugino sector are discussed in [44, 45].

To the best of our knowledge no study has been done to find non-SM type Higgs boson 
signals within the NMSSM at the LHeC. In our analysis, we assumed the second intermediate Higgs boson is the SM-type ( $h_{2}$-SM scenario). This, of course, refers to the mass, coupling ratios and signal-strength from the recent LHC results. The lightest non-SM Higgs boson, $h_{1}$, offers itself to be looked for at the upcoming LHeC. We identify two main production processes, namely the neutral current one $e p \rightarrow e h_{1} q$ and the charged current one $e p \rightarrow \nu h_{1} q$. We are particularly motivated by the possible branching ratio enhancement in the $b$-quark decay mode, i.e., $h_{1} \rightarrow b \bar{b}$. Finally the reconstructed invariant mass of the two $b$-tagged jets ensures the evidence of the non-SM like Higgs boson.

The plan of this paper is as follows. In the next section we will describe briefly the NMSSM model. In Sec.2, we will randomly vary the NMSSM model parameters and identify the allowed parameter space consistent with most up-to-date theoretical, phenomenological and experimental constraints. For the allowed model space, we then estimate the number of non-SM type Higgs boson signal events, $e p \rightarrow e h_{1} q$ and $e p \rightarrow \nu h_{1} q$ with the decay channel $h_{1} \rightarrow b \bar{b}$ and identify few high event-rated benchmark points to carry out the phenomenological analysis in Sec.3. In doing so, we estimate all the reducible and irreducible SM backgrounds for both of the signal channels under considerations. In Sec.4, we carry out a simple cut-based optimization to isolate the Higgs boson signals in both the channels. We summarize our findings in Sec.5.

\section{The NMSSM models}

The NMSSM model has been described in many reviews [11, 12, 13, 14, 15. However, for completeness let us mention the part relevant for our analysis (we will follow [15]).

The general NMSSM contains the MSSM superfields with most general renormalizable couplings as in the MSSM superpotential. There is, however, one additional gauge singlet chiral superfield $\widehat{S}$.

The Higgs superpotential $W_{\text {Higgs }}$ reads

$$
W_{\text {Higgs }}=(\mu+\lambda \widehat{S}) \widehat{H}_{u} \cdot \widehat{H}_{d}+\xi_{F} \widehat{S}+\frac{1}{2} \mu^{\prime} \widehat{S}^{2}+\frac{\kappa}{3} \widehat{S}^{3}
$$

where $\lambda, \kappa$ are dimensionless Yukawa couplings. The bi-linear $\mu, \mu^{\prime}$ terms are the supersymmetric mass terms, and $\xi_{F}$ with mass-dimension two is the supersymmetric tadpole term.

Assuming R-parity and CP-conservation (scenarios without these requirements have been studied in [48] and [22]) the corresponding soft supersymmetry breaking terms, $\mathcal{L}_{\text {soft }}$ are the following:

$$
\begin{aligned}
-\mathcal{L}_{\text {soft }}= & m_{H_{u}}^{2}\left|H_{u}\right|^{2}+m_{H_{d}}^{2}\left|H_{d}\right|^{2}+m_{S}^{2}|S|^{2}+m_{Q}^{2}\left|Q^{2}\right|+m_{U}^{2}\left|U_{R}^{2}\right| \\
& +m_{D}^{2}\left|D_{R}^{2}\right|+m_{L}^{2}\left|L^{2}\right|+m_{E}^{2}\left|E_{R}^{2}\right| \\
& +\left(h_{u} A_{u} Q \cdot H_{u} U_{R}^{c}-h_{d} A_{d} Q \cdot H_{d} D_{R}^{c}-h_{e} A_{e} L \cdot H_{d} E_{R}^{c}\right. \\
& \left.+\lambda A_{\lambda} H_{u} \cdot H_{d} S+\frac{1}{3} \kappa A_{\kappa} S^{3}+m_{3}^{2} H_{u} \cdot H_{d}+\frac{1}{2} m_{S}^{\prime 2} S^{2}+\xi_{S} S+\text { h.c. }\right),
\end{aligned}
$$

where all the parameters have the standard meanings.

The dimensionful supersymmetric parameters $\mu, \mu^{\prime}$ and $\xi_{F}$ ((with mass dimension two) in the superpotential and the associated soft SUSY breaking parameters $m_{3}^{2}, m_{S}^{\prime 2}$ and $\xi_{S}$ (with mass dimension three) have to be of the order of the weak or SUSY breaking scale. 
In general, these terms are non-vanishing, however a simplified version requiring scale invariance leads to $\mu=\mu^{\prime}=\xi_{F}=0$ and the superpotential takes the simple form

$$
W_{\text {scale-invariant }}=\lambda \widehat{S} \widehat{H}_{u} \cdot \widehat{H}_{d}+\frac{\kappa}{3} \widehat{S}^{3}
$$

together with the parameters $m_{3}^{2}, m_{S}^{\prime 2}$ and $\xi_{S}$ in (2) also set to zero. An effective $\mu$-term of the order of weak scale is generated from the vev $s$ of $\widehat{S}$ :

$$
\mu_{\mathrm{eff}}=\lambda s
$$

From the supersymmetric gauge interactions, the $F$-term and the soft supersymmetry breaking terms one can obtain the Higgs potential:

$$
\begin{aligned}
V_{\text {Higgs }}= & \left|\lambda\left(H_{u}^{+} H_{d}^{-}-H_{u}^{0} H_{d}^{0}\right)+\kappa S^{2}+\mu^{\prime} S+\xi_{F}\right|^{2} \\
& +\left(m_{H_{u}}^{2}+|\mu+\lambda S|^{2}\right)\left(\left|H_{u}^{0}\right|^{2}+\left|H_{u}^{+}\right|^{2}\right)+\left(m_{H_{d}}^{2}+|\mu+\lambda S|^{2}\right)\left(\left|H_{d}^{0}\right|^{2}+\left|H_{d}^{-}\right|^{2}\right) \\
& +\frac{g_{1}^{2}+g_{2}^{2}}{8}\left(\left|H_{u}^{0}\right|^{2}+\left|H_{u}^{+}\right|^{2}-\left|H_{d}^{0}\right|^{2}-\left|H_{d}^{-}\right|^{2}\right)^{2}+\frac{g_{2}^{2}}{2}\left|H_{u}^{+} H_{d}^{0 *}+H_{u}^{0} H_{d}^{-*}\right|^{2} \\
& +m_{S}^{2}|S|^{2}+\left(\lambda A_{\lambda}\left(H_{u}^{+} H_{d}^{-}-H_{u}^{0} H_{d}^{0}\right) S+\frac{1}{3} \kappa A_{\kappa} S^{3}+m_{3}^{2}\left(H_{u}^{+} H_{d}^{-}-H_{u}^{0} H_{d}^{0}\right)\right. \\
& \left.+\frac{1}{2} m_{S}^{\prime 2} S^{2}+\xi_{S} S+\text { h.c. }\right)
\end{aligned}
$$

where $g_{1}$ and $g_{2}$ are $U(1)_{Y}$ and $S U(2)$ gauge couplings, respectively.

The physical neutral Higgs fields (with index $\mathrm{R}$ for the CP-even, index I for the CP-odd states) are obtained by expanding the full scalar potential (5) around the real neutral vevs $v_{u}$, $v_{d}$ and $s$ :

$$
H_{u}^{0}=v_{u}+\frac{H_{u R}+i H_{u I}}{\sqrt{2}}, \quad H_{d}^{0}=v_{d}+\frac{H_{d R}+i H_{d I}}{\sqrt{2}}, \quad S=s+\frac{S_{R}+i S_{I}}{\sqrt{2}} ;
$$

where the vevs have to be obtained from the minima of

$$
\begin{aligned}
V_{\text {Higgs }}= & \left(-\lambda v_{u} v_{d}+\kappa s^{2}+\mu^{\prime} s+\xi_{F}\right)^{2}+\frac{g_{1}^{2}+g_{2}^{2}}{8}\left(v_{u}^{2}-v_{d}^{2}\right)^{2} \\
& +\left(m_{H_{u}}^{2}+(\mu+\lambda s)^{2}\right) v_{u}^{2}+\left(m_{H_{d}}^{2}+(\mu+\lambda s)^{2}\right) v_{d}^{2} \\
& +m_{S}^{2} s^{2}-2 \lambda A_{\lambda} v_{u} v_{d} s+\frac{2}{3} \kappa A_{\kappa} s^{3}-2 m_{3}^{2} v_{u} v_{d}+m_{S}^{\prime 2} s^{2}+2 \xi_{S} s,
\end{aligned}
$$

The minimization of (7) with respect the the three vevs and the proper electroweak symmetry breaking (generating the correct $Z$-boson mass) leads to the following input parameters:

$$
\lambda, \kappa, A_{\lambda}, A_{\kappa}, \tan \beta, \mu_{\mathrm{eff}},
$$

to which one has to add the (in the convention $\mu=0$ ) five parameters of the NMSSM

$$
m_{3}^{2}, \mu^{\prime}, m_{S}^{\prime 2}, \xi_{F} \text { and } \xi_{S}
$$

The tree level Higgs mass matrices are obtained by expanding the full scalar potential (5) around the real neutral vevs $v_{u}, v_{d}$ and $s$ as in (6). The elements of the $3 \times 3$ CP-even mass 


\begin{tabular}{||c||c|c||}
\hline Parameters & Min & Max \\
\hline \hline$\lambda$ & 0.001 & 0.7 \\
$\kappa$ & 0.001 & 0.7 \\
$A_{\lambda}$ & 100.0 & 2500.0 \\
$A_{\kappa}$ & -2500.0 & 100.0 \\
$\tan \beta$ & 1.5 & 60.0 \\
$\mu_{e f f}$ & 100.0 & 500.0 \\
$M_{1}$ & 50.0 & 400.0 \\
$M_{2}$ & 50.0 & 500.0 \\
$m_{\tilde{q}}$ & 300.0 & 1500.0 \\
$A_{t}=A_{b}$ & -4000.0 & 1000.0 \\
$M_{A}$ & 100.0 & 500.0 \\
$M_{P}$ & 100.0 & 3000.0 \\
\hline \hline
\end{tabular}

Table 1: The minimum and maximum values of varied NMSSM parameters. The following parameters remain fixed: $M_{3}=1800.0 \mathrm{GeV}$ (this allows the gluino mass $m_{\tilde{g}}$ to be above the mass limits from recent LHC-run2); $m_{\tilde{\ell}}=300.0 \mathrm{GeV}$ (for all three generation as well as left and right state) and $A_{\tau}=A_{e}=A_{\mu}=1500.0 \mathrm{GeV}$. Here $M_{A}\left(M_{P}\right)$ is the Doublet(Singlet) component of the CP-odd Higgs mass matrices.

matrix $\mathcal{M}_{S}^{2}$ are conveniently written in the basis $\left(H_{d R}, H_{u R}, S_{R}\right)$ after the elimination of $m_{H_{d}}^{2}$, $m_{H_{u}}^{2}$ and $m_{S}^{2}$.

The basis for the elements of the $3 \times 3 \mathrm{CP}$-odd mass matrix $\mathcal{M}_{P}^{\prime 2}$ are $\left(H_{d I}, H_{u I}, S_{I}\right)$. Dropping the Goldstone mode, in the remaining $2 \times 2 \mathrm{CP}$-odd mass matrices one can use the doublet $\left(M_{A}\right)$ and singlet component $\left(M_{P}\right)$ mass parameters as inputs together with the $\mu_{e f f}$.

Our model under consideration is not the $Z_{3}$ invariant NMSSM, but rather a general phenomenological NMSSM. However, by setting: $m_{3}^{2}=m_{S}^{\prime 2}=\xi_{S}=\mu=\mu^{\prime}=\xi_{F}=0$ in the general phenomenological NMSSM, one recovers the $Z_{3}$ invariant NMSSM.

\section{The NMSSM parameter spaces}

We used the package NMSSMTools 5.0.1 [49] to obtain the Sparticle spectrum, decay branching ratios and various low energy observables.

We randomly scanned approximately $10^{6}$ points. The varied parameters and their ranges are 3 tabulated in Table 1 .

For each randomly generated parameter spaces, we invoke the following constraints:

Perturbative bounds: We first imposed, $\lambda^{2}+\kappa^{2} \lesssim(0.7)^{2}$ [52] and if not satisfied we discard the parameter space and generate the next random model space.

Dark Matter relic density: We required that the lightest neutralino relic density will be: $0.107<\Omega_{\tilde{\chi}_{1}^{0}} h^{2}<0.131$, consistent with the Planck measurement [39] within standard cosmology. The estimated relic density $\left(\Omega_{\tilde{\chi}_{1}^{0}} h^{2}\right)$ as a function of the $m_{\tilde{\chi}_{1}^{0}}$ has been shown in the left panel of Fig.1 with constraining only the upper limits, i.e., $\Omega_{\tilde{\chi}_{1}^{0}} h^{2}<0.131$. The green-marked points within the upper and lower strips are consistent with the directdetection and indirect-detection bounds (in the legends termed as: "All-DM").

The NMSSMTools 5.0.1 is interfaced with micrOMEGAs v4.3 [50, 51] to estimate the observed dark matter relic density, their direct detection, and indirect detection limits. It

\footnotetext{
${ }^{3}$ All the masses and mass parameters in our analysis are in $\mathrm{GeV}$.
} 

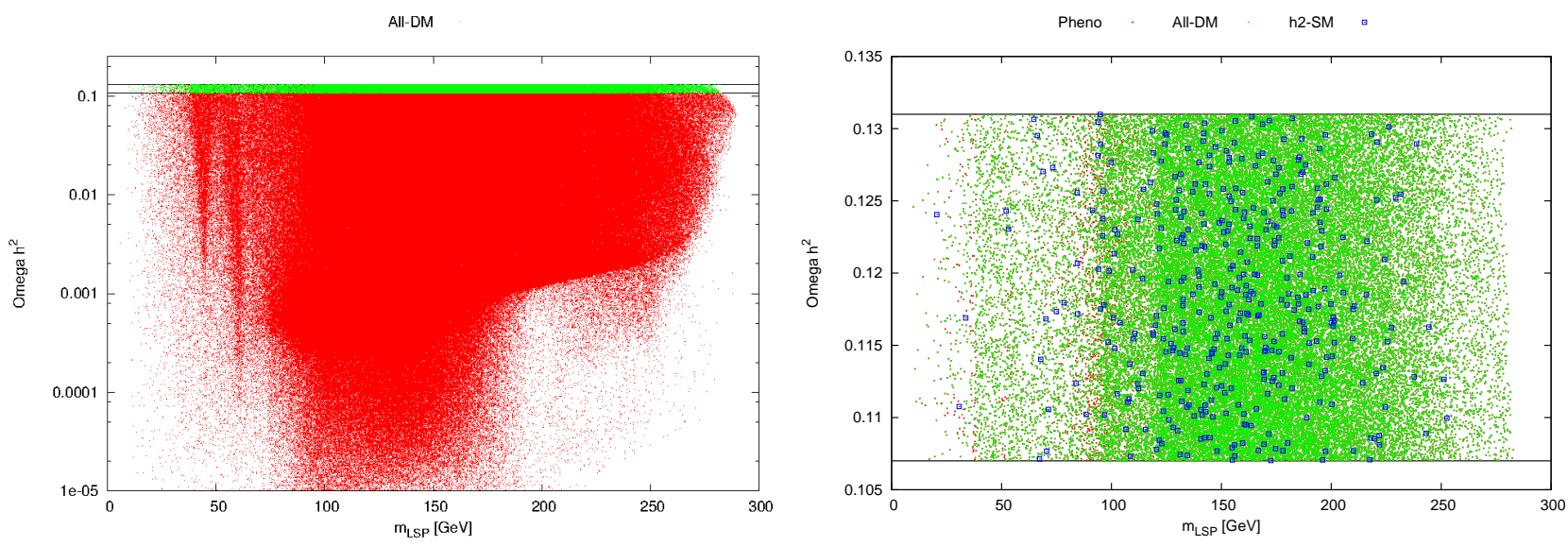

Figure 1: Left-panel: The Dark matter relic density $\left(\Omega_{\tilde{\chi}_{1}^{0}} h^{2}\right)$ as a function of lightest neutralino $\operatorname{masses}\left(m_{\tilde{\chi}_{1}^{0}}\right)$ within the standard cosmology. The two lines represent the upper $\left(\Omega_{\tilde{\chi}_{1}^{0}} h^{2}=0.131\right)$ and lower $\left(\Omega_{\tilde{\chi}_{1}^{0}} h^{2}=0.107\right)$ bounds from the Planck measurements [39]. The first deep around $45 \mathrm{GeV}$ is due to the Z-boson exchange (annihilation diagram) and the second around $63 \mathrm{GeV}$, from the Higgs-boson $\left(h_{2}-\mathrm{SM}\right)$ exchange annihilation within the NMSSM parameter spaces. The green point within the strips satisfy the direct and indirect Dark Matter searches results and termed as "All-DM". Right-panel: we only show the relic-density allowed parameter space together with various other constraints (in the legends), see the text for details.
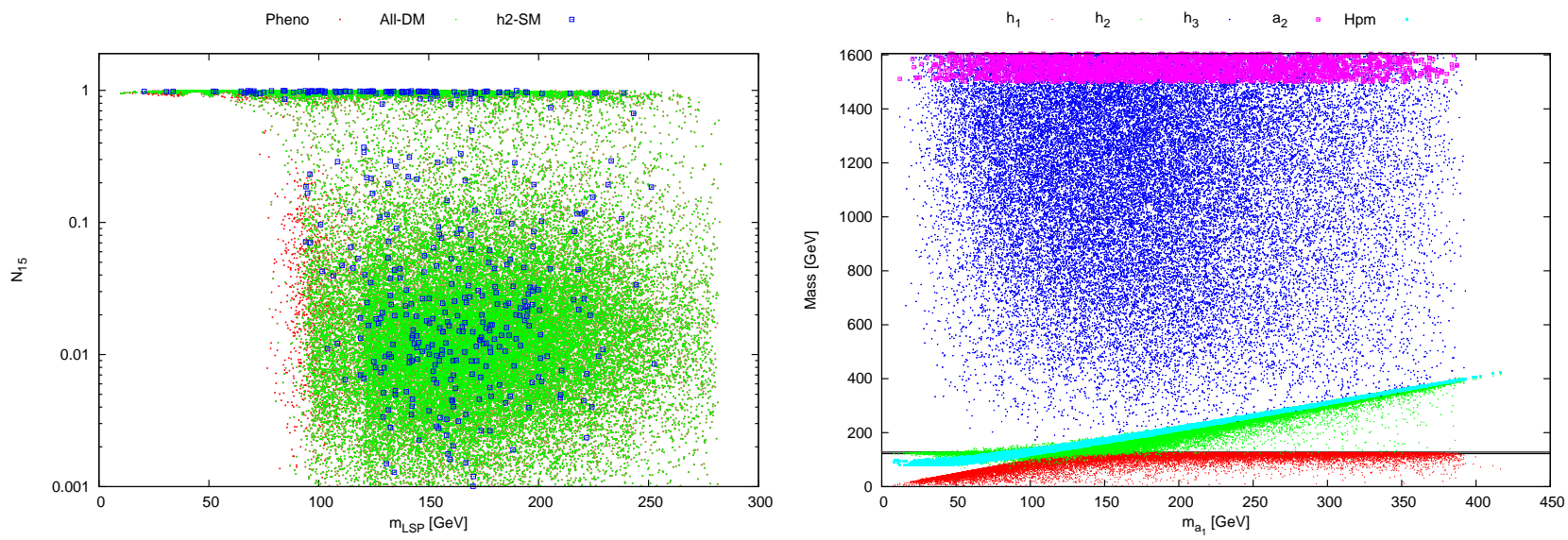

Figure 2: Left-panel: The Singlet component $\left(N_{15}\right)$ of the lightest neutralino $\left(\tilde{\chi}_{1}^{0}\right)$ consistent with the dark matter relic density $\left(\Omega_{\tilde{\chi}_{1}^{0}} h^{2}\right)$ and various other constraints (see text for details). Right-panel: The masses of the Higgs boson masses, $h_{1}, h_{2}, h_{3}, a_{2}$ and $h^{ \pm}$("Hpm") as a function of the lightest CP-odd Higgs bosons masses $\left(m_{a_{1}}\right)$. The mass of the $a_{2}$ is shown up to 1600.0 GeV, extends, however, even beyond this point in this allowed NMSSM model parameter spaces.

is to be noted that the standard and non-standard cosmological implication in the dark matter relic density has been analyzed within the NMSSM in [27, 40, 41]. 

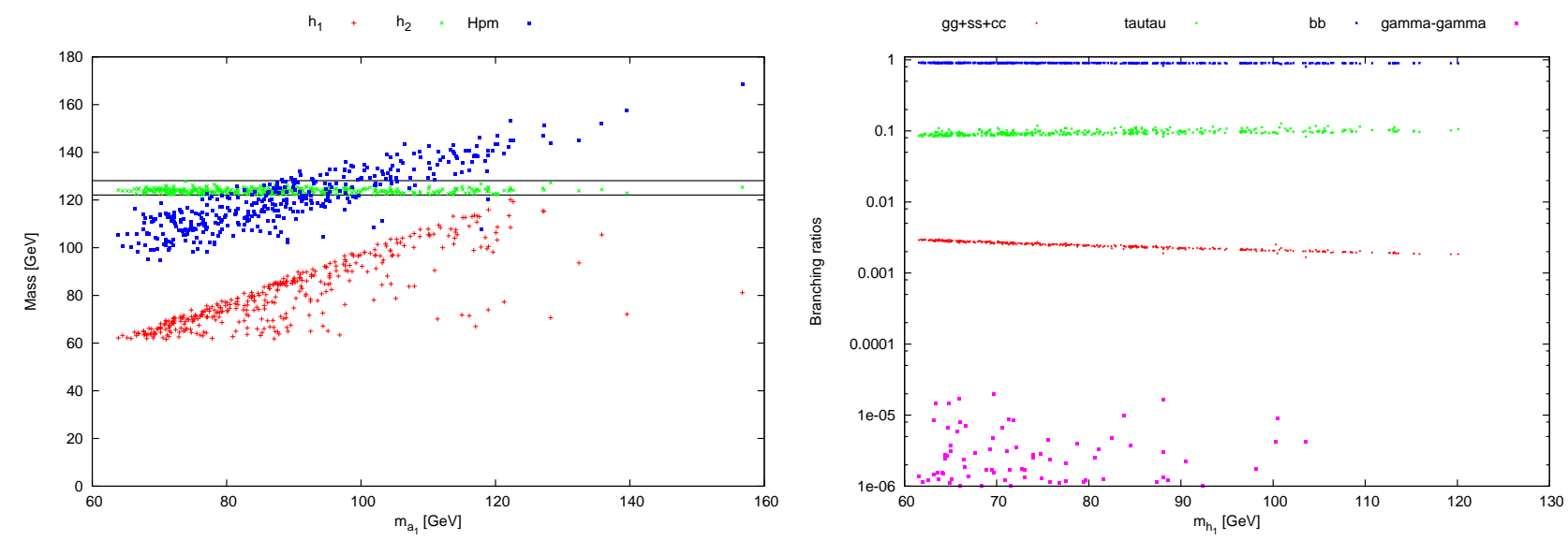

Figure 3: Left-panel: The Higgs boson masses, $h_{1}, h_{2}$, and $h^{ \pm}$as a function of the lightest CP-odd Higgs bosons mass $\left(m_{a_{1}}\right)$ for $h_{2}$-SM scenario and consistent with all other constraints (see details in the text). In the right panel, we show the branching ratio of the $h_{1}$ in different channels and it is clear that in large region of allowed parameter space the branching ratio of $h_{1} \rightarrow b \bar{b}$ is above $90 \%$.

\begin{tabular}{||c||c|c||c||c|c||}
\hline Parameters & Min & Max & Parameters & Min & Max \\
\hline \hline$\kappa_{W}$ & 0.81 & 0.99 & $\mu_{V B F}^{\tau \tau}$ & 0.50 & 2.10 \\
$\kappa_{t}$ & 0.99 & 1.89 & $\mu_{g g F}^{\tau \tau}$ & -0.20 & 2.20 \\
$\left|\kappa_{\gamma}\right|$ & 0.72 & 1.10 & $\mu_{V V}^{b b}$ & 0.00 & 2.00 \\
$\left|\kappa_{g}\right|$ & 0.61 & 1.07 & $\mu_{t t H}^{b b}$ & -0.90 & 3.10 \\
$\left|\kappa_{\tau}\right|$ & 0.65 & 1.11 & $\mu_{V Z F}^{W W}$ & 0.40 & 2.00 \\
$\left|\kappa_{b}\right|$ & 0.25 & 0.89 & $\mu_{g g F}^{Z Z}$ & 0.51 & 1.81 \\
$B r\left(h_{S M} \rightarrow\right.$ inv. $)$ & & 0.25 & $\mu_{V \gamma}^{\gamma \gamma}$ & 0.30 & 2.30 \\
& & & $\mu_{g g F}^{\gamma \gamma}$ & 0.66 & 1.56 \\
\hline \hline
\end{tabular}

Table 2: The couplings $(\kappa)$ and signal strength $(\mu)$ have been allowed within $2 \sigma$ ranges (except $\left.\kappa_{W}\right)$ from the combined ATLAS and CMS measurements [54], following Table 17 (upper panel) and Table 8 respectively.

Higgs bounds: We demand that the intermediate Higgs boson $\left(h_{2}\right)$ should be SM-like and its mass should be within the range of $125.09 \mathrm{GeV}<m_{h_{2}}<128.09 \mathrm{GeV}$ (taken into consideration the $3 \mathrm{GeV}$ error in theoretical estimates). Its coupling ratios to other SM particles should also be consistent with the LHC-run1 ATLAS and CMS combined study [53, 54. The allowed coupling ratios and the signal strengths considered in our analysis has been tabulated in Table 2. We have also taken the constraint on the invisible branching ratio: $B R\left(h_{S M} \rightarrow\right.$ invisible $) \lesssim 0.25$ [55, 56, 4]. Furthermore, we required $m_{h^{ \pm}}>80.0 \mathrm{GeV}$.

LEP bounds: Direct SUSY searches at the LEP have set bounds on superpartners, in particular the lighter chargino should satisfy $m_{\tilde{\chi}_{1}^{ \pm}}>103.5 \mathrm{GeV}$. The other one refers to the $Z$ invisible width and should satisfy $\Gamma_{Z}^{\text {inv }}<2 \mathrm{MeV}$ at 95\% C.L. [58]. When the decay channel into $\tilde{\chi}_{1}^{0} \tilde{\chi}_{1}^{0}$ opens, this width may exceed the experimental value 5 .

\footnotetext{
${ }^{4}$ The SM-like Higgs boson invisible decay within the NMSSM has been studied recently in [57].

${ }^{5}$ A light Higgs would significantly affect the muon anomalous magnetic moment $a_{\mu}=\left(g_{\mu}-2\right) / 2$, whose most
} 
B physics bounds: The rare decays of $B$ meson, such as $B_{s} \rightarrow \mu^{+} \mu^{-}, B^{+} \rightarrow \tau^{+} \nu$, and $B_{s} \rightarrow X_{s} \gamma$ lead to the flavor constraints. In our analysis, we set the recent experimental results at $95 \%$ C.L.: $1.7 \times 10^{-9}<\mathrm{BR}\left(B_{s} \rightarrow \mu^{+} \mu^{-}\right)<4.5 \times 10^{-9}\left[60\right.$, $0.85 \times 10^{-4}<$ $\mathrm{BR}\left(B^{+} \rightarrow \tau^{+} \nu\right)<2.89 \times 10^{-4}$ [61], and $2.99 \times 10^{-4}<\mathrm{BR}\left(B_{s} \rightarrow X_{s} \gamma\right)<3.87 \times 10^{-4}[60$ ].

Sparticle masses: We have set the following lower bounds from the superparticle masses following [62]: $m_{\tilde{g}} \gtrsim 1600.0 \mathrm{GeV}, m_{\tilde{t}_{1}} \gtrsim 95.0 \mathrm{GeV}, m_{\tilde{b}_{1}} \gtrsim 325.0 \mathrm{GeV}, m_{\tilde{q}_{L}} \gtrsim 600.0$ $\mathrm{GeV}, m_{\tilde{\ell}_{L}} \gtrsim 100.0 \mathrm{GeV}, \tilde{m}_{\nu_{L}} \gtrsim 90.0 \mathrm{GeV}$ and $m_{\tilde{\tau}_{1}} \gtrsim 87.0 \mathrm{GeV}$.

If the randomly generated NMSSM model space satisfy all the above constraints, we consider them for further phenomenological studies. In the left-panel of Fig! 1 we calculated $\Omega_{\tilde{\chi}_{1}^{0}} h^{2}$ using the micrOMEGAs as a function the mass of the cold-dark matter candidate $\left(m_{\tilde{\chi}_{1}^{0}}\right)$. All the points satisfy the upper bounds coming from the recent Planck measurements [39] and the upper and lower bounds are 0.131 and 0.107 , respectively referring to the standard cosmological scenario. Within this strips, the green point satisfy the direct and indirect Dark Matter searches which we term as "All-DM" . The lightest neutralino annihilation would occur via the Z-boson exchange diagram - this shows a dip around the $M_{Z} / 2$, i.e, $45 \mathrm{GeV}$. This annihilation rate would get enhanced via the SM-Higgs boson $\left(h_{2}\right)$ exchange diagram and this leads to another dip around $m_{h_{2}} / 2$, i.e., $63 \mathrm{GeV}$.

In the right panel of Fig, 1, we invoke other constraints discussed above. If the constraints on the Sparticles masses and B-physics and other phenomenological limits are satisfied, we indicated it by "Pheno"; and if $h_{2}$-SM type scenario is satisfied, we indicate it by " $h 2-S M "$. All the constraints are imposed cumulatively. Finally, the $h 2-\mathrm{SM}$ model is interesting per se to look for as it is an unusual scenario.

In the left-panel of Fig,2, we displayed the singlino component of the lightest neutralino, i.e., $N_{15}$ as a function of $m_{\tilde{\chi}_{1}^{0}}$ with all the constraints mentioned in the legend. This shows that lightest neutralino with $m_{\tilde{\chi}_{1}^{0}} \gtrsim 80 \mathrm{GeV}$ are more favorable with the $h_{2}$-SM type scenario. It can be seen that the singlino domination would occur mostly between $25 \mathrm{GeV}$ and $250 \mathrm{GeV}$, while non-singlino type $\tilde{\chi}_{1}^{0}$ would also be possible (would go up to $1 \%$ ) but the mass ranges are rather squeezed ranging between $100-250 \mathrm{GeV}$.

In the right-panel of Fig,2, we showed the masses of all the Higgs bosons satisfying all constraints. It turns out that the heavy CP-odd Higgs boson mass (we showed it up to 1600.0 $\mathrm{GeV}$ ) is quite heavy, as it mainly depends upon the values of $M_{P}$.

In the left-panel of Fig 3 we showed all the Higgs boson masses as a function of $m_{a_{1}}$. However, in comparison to the right-panel of Fig 2 here we additionally imposed the "All-DM", "Pheno" and "h2-SM" criterion. This particular parameter spaces are of our phenomenological interest. In the right-panel of Fig. 3 , we show the decay branching ratios of the non-SM type $h_{1}$ in the light flavor quark mode: $h_{1} \rightarrow g g+c \bar{c}+s \bar{s}$, tau-lepton: $h_{1} \rightarrow \tau \bar{\tau}, b$-quark: $h_{1} \rightarrow b \bar{b}$ and the much suppressed two-photon decay: $h_{1} \rightarrow \gamma \gamma$.

For all these points we estimated the event rates for the two signal processes under consideration. We will describe the details in the following section.

accurate measurement comes from the E821 experiment [59]. Having the large theoretical uncertainties with the measurements, we have not consider this constraints in our numerical scan and subsequent analysis. 


\section{Numerical Analysis}

In our analysis we consider the $h_{2}$-SM scenario following Fig. 3, such that the non-SM like $h_{1}$ is light enough to have some appreciable production rates at $\mathrm{LHeC}$.

In this section, we first describe the main two different production mechanism of Higgs bosons at the LHeC collider. These are the neutral and charged current production, which lead to the $e+3 \mathrm{j}$ and $\mathbb{E}_{T}+3 \mathrm{j}$ signals, respectively. As a result we will have two different sets of SM backgrounds which we will address in the subsequent section.

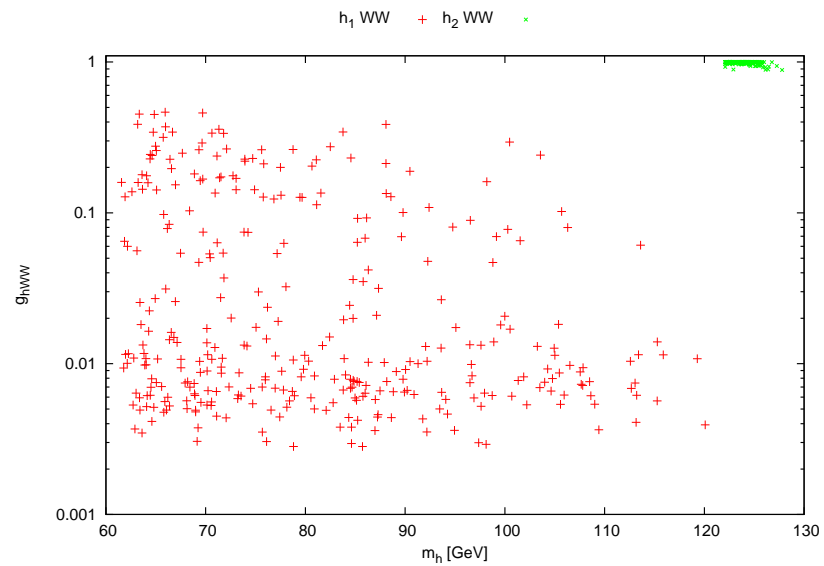

Figure 4: The coupling $g_{h W W}$ (for $h=h_{1}$ and $h_{2}$ ) is shown as a function of the Higgs boson mass. The top right corner (with populated green points) shows the masses of the $h_{2}$ and the corresponding coupling constraints (that we imposed following Table 2) from the recent LHC measurements.
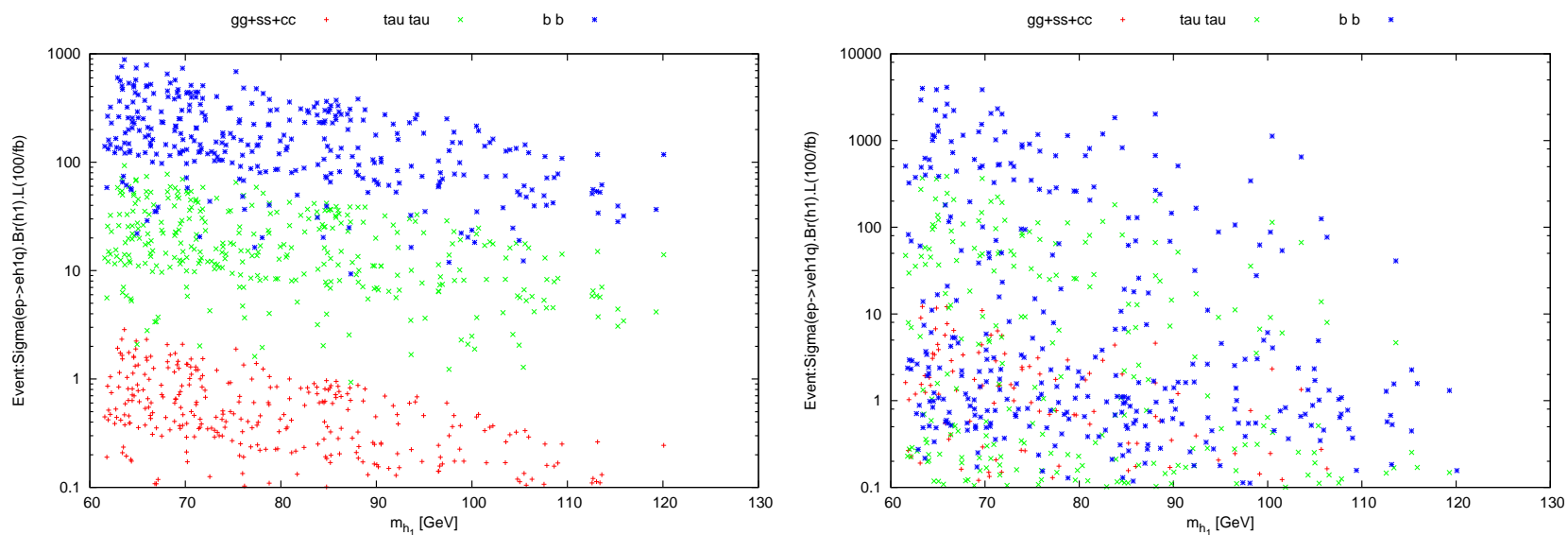

Figure 5: Number of event in $e+3$-jets (left-panel) and $\mathbb{F}_{T}+3$-jets (right-panel) channels at LHeC with integrated luminosity of $100 \mathrm{fb}^{-1}$. 

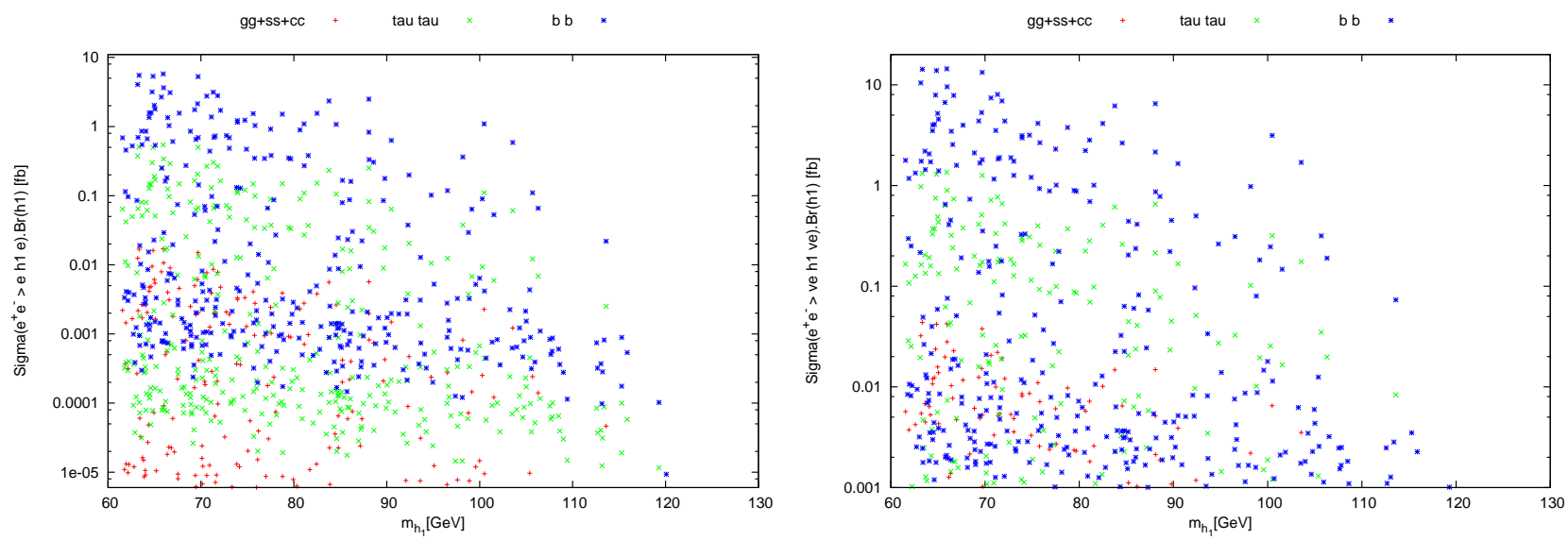

Figure 6: The cross-section multiplied with the Higgs branching ratios in units of $[f b]$ in the $e^{+} e^{-}$LEP collider with center of mass energy $209 \mathrm{GeV}$, for the $\sigma\left(e^{+} e^{-} \rightarrow e^{+} h_{1} e^{-}\right)$and $\sigma\left(e^{+} e^{-} \rightarrow \nu_{e} h_{1} \bar{\nu}_{e}\right)$ in the left and right panel respectively.
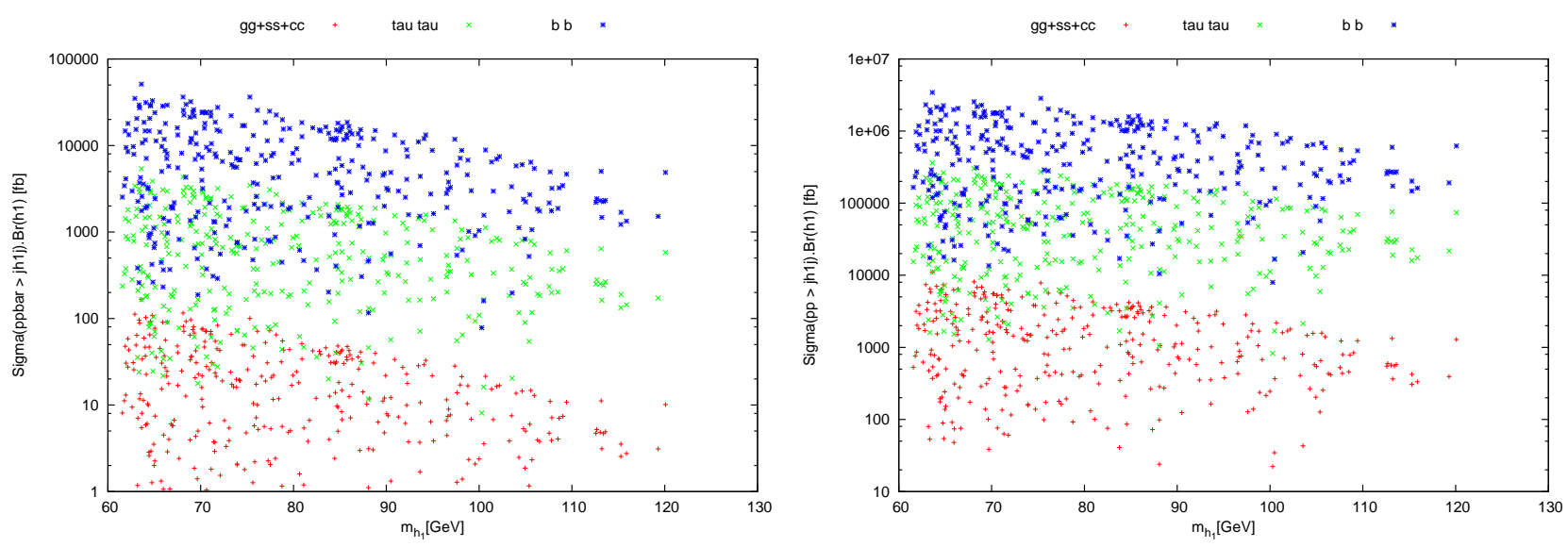

Figure 7: Left-panel: The cross-section with Higgs branching ratios in units of [fb] at the Tevatron $\left(\sigma\left(p \bar{p} \rightarrow j h_{1} j\right)\right)$ with center of mass energy $1.96 \mathrm{TeV}$. Right-panel: $\sigma\left(p p \rightarrow j h_{1} j\right)$ at LHC collider with center of mass energy $14 \mathrm{TeV}$, where $j=\mathrm{u}, \mathrm{d}, \mathrm{c}, \mathrm{s}, \mathrm{b}, \mathrm{g}$ and their charge-conjugation.

\subsection{Higgs bosons signals}

In our analysis, we consider the leading production processes via the neutral current as well as charged current. As we consider an ep-collison, the neutral (charged) current gives charged (neutral) lepton, electron (neutrino) in the final states. Generally, the charged current production cross-sections are larger than the neutral currents as shown in Fig 5 which is mainly due to the isospin couplings. The production processes of the Higgs bosons are : $e h_{1} q$ and $\nu_{e} h_{1} q$, 6 where $q$ (or its charge conjugated version) is the light parton, i.e., $u, d, s, c$ or $g$.

\footnotetext{
${ }^{6}$ Please recall that we are working in the $h_{2}$-SM scenario. This is to say that the second CP-even Higgs boson is the SM-type consistent with all the couplings and observables and in particular compatible with the recent Higgs discovery at the LHC experiments.
} 
The Higgs boson production in our analysis is mainly dominated by the t-channel vectorboson fusion (VBF) processes.

The couplings (the neutral and charged gauge-boson fusion vertex) relevant at LHeC are the following:

$$
g_{h_{1} Z Z}=\frac{g_{1}^{2}+g_{2}^{2}}{\sqrt{2}}\left(v_{d} S_{11}+v_{u} S_{12}\right), \quad g_{h_{1} W W}=\frac{g_{2}^{2}}{\sqrt{2}}\left(v_{d} S_{11}+v_{u} S_{12}\right)
$$

Here $S_{11}$ and $S_{12}$ are the mixing parameters in the singlet-doublet Higgs mixing matrices. The $v_{u}$ and $v_{d}$ are the up-type and down-type Higgs doublet vevs and $g_{1}$ and $g_{2}$ are the $U(1)_{Y}$ and $S U(2)_{L}$ gauge couplings. We have plotted the allowed couplings, $g_{h_{1} W W}$, (using red color plus points) complying all the constraints discussed earlier in Fig 4 (see the caption for details). The green points in the top right corner are for $g_{h_{2} W W}$ and the masses and the corresponding coupling values consistent with the coupling ratios for the $h_{2}$-SM scenario, following Table 2.

It is to be noted that the same couplings given in Eq10 are also responsible for the Higgs boson production (t-channel VBF) at the LEP and Hadron colliders. We would like to estimate the event rates for this kind of signal at the large electron positron (LEP) colliders and also at the hadron colliders, like the recently closed Tevatron and presently operating LHC.

We find that the exact signal hard-processes at LHeC under considerations are not possible at LEP, Tevatron and LHC. However, we estimated the closest processes that we could have in these three colliders. At these colliders, we have estimated the event rates (in $f b$ ) with inclusion of Higgs branching ratios at the $e^{+} e^{-}$LEP collider with center of mass energy $209 \mathrm{GeV}$, for the $\sigma\left(e^{+} e^{-} \rightarrow e^{+} h_{1} e^{-}\right)$and $\sigma\left(e^{+} e^{-} \rightarrow \nu_{e} h_{1} \bar{\nu}_{e}\right)$ processes in Fig $[6$ in the left-panel and right-panel respectively.

In hadron colliders the most closet process is $\sigma\left(p p, p \bar{p} \rightarrow j h_{1} j\right)$, with $j=u, d, c, s, b, g$ and their charge-conjugation. The estimated event rates (in $f b$ ) at Tevatron and LHC with center of mass energy $1.96 \mathrm{TeV}$ and $14 \mathrm{TeV}$, is shown in Fig,7, in the left-panel and right-panel respectively. The LHC collider is presently operating and the number of events rates are substantial. In spite of the huge SM backgrounds contamination, it would be worth to look for this non-standard Higgs signal in this ongoing machine.

We see from the right-panel of Fig 3 that $h_{1}$ is dominantly decaying into $b \bar{b}$. So our signals in both channels contain three jets (one is forward light flavored and two central $b$-tagged) and an electron (missing transverse energy) in case of neutral (charged) current.

We estimated the parton level signal cross sections using the MadGraph v 2.4.3 [63]. The allowed NMSSM model parameter spaces from the NMSSMTools 5.0.1 [49] are written in SLHA format and fed to MadGraph v 2.4.3 [63]. The Branching ratios (BRs) of the Higgs boson in all the decay modes is estimated by using NMHDECAY [49].

To obtain the cross sections at the LHeC [32, 64, 65, 66, 67], we consider an electron beam, of energy $E_{e^{-}}=60 \mathrm{GeV}$ and a proton beam of energy $E_{p}=7000 \mathrm{GeV}$, corresponding to a center-of-mass energy of approximately $\sqrt{s}=1.296 \mathrm{TeV}$.

To estimate the signal event rates at parton level we applied the following basic preselections:

$$
p_{T}^{q, e}>10 \mathrm{GeV}, \quad \eta^{q, e}<5.0 \quad \Delta R(q q, q e)>0.2
$$

with $\Delta R^{2}=\Delta \eta^{2}+\Delta \phi^{2}$, where $\eta$ and $\phi$ are the pseudo-rapidity and azimuthal angle, respectively. We take $m_{t}=173.3 \mathrm{GeV}$ as the top-quark pole mass. 


\begin{tabular}{|c|c|c|c|c|c|c|c|c|c|c|}
\hline Benchmark Points & $e 1$ & $e 2$ & $e 3$ & $e 4$ & $e 5$ & $\nu 1$ & $\nu 2$ & $\nu 3$ & $\nu 4=e 5$ & $\nu 5$ \\
\hline $\bar{\lambda}$ & 0.241 & 0.168 & 0.171 & 0.237 & 0.384 & 0.208 & 0.263 & 0.296 & 0.384 & 0.498 \\
\hline$\kappa$ & 0.371 & 0.0567 & 0.0324 & 0.0384 & 0.0152 & 0.0577 & 0.143 & 0.147 & 0.0152 & 0.291 \\
\hline $\tan \beta$ & 56.71 & 48.62 & 56.32 & 56.27 & 4.41 & 3.81 & 4.95 & 5.15 & 4.41 & 6.18 \\
\hline$A_{\lambda}(\mathrm{GeV})$ & 974.0 & 1007.8 & 1230.8 & 964.1 & 1222.4 & 1130.3 & 1105.8 & 1078.3 & 1222.4 & 1107.8 \\
\hline$A_{\kappa}(\mathrm{GeV})$ & -1139.2 & -1105.6 & -1214.2 & -1108.3 & -1062.3 & -1230.3 & -835.9 & -803.6 & -1062.3 & -1732.7 \\
\hline$\mu_{\text {eff }}$ & 466.4 & 272.9 & 297.2 & 454.6 & 381.1 & 392.6 & 374.5 & 342.7 & 381.1 & 193.8 \\
\hline$M_{1}(\mathrm{GeV})$ & 274.6 & 347.5 & 241.7 & 272.5 & 335.3 & 173.3 & 146.5 & 172.7 & 335.3 & 112.1 \\
\hline$M_{2}(\mathrm{GeV})$ & 293.4 & 482.3 & 462.5 & 277.5 & 352.3 & 283.9 & 257.3 & 495.3 & 352.3 & 430.7 \\
\hline$M_{\tilde{q}}$ & 1010.4 & 941.9 & 700.6 & 1036.6 & 734.0 & 807.1 & 812.9 & 772.5 & 734.0 & 762.0 \\
\hline$\overline{A_{t}}=A_{b}(\mathrm{GeV})$ & -2661.8 & -1695.4 & -1735.6 & -2679.4 & -1585.1 & -1946.3 & -1883.7 & -1676.3 & -1585.1 & -1894.7 \\
\hline$M_{A}(\mathrm{GeV})$ & 316.9 & 282.2 & 239.8 & 319.9 & 125.6 & 102.9 & 106.9 & 131.7 & 125.6 & 134.5 \\
\hline$M_{P}(\mathrm{GeV})$ & 2015.5 & 1650.2 & 1995.9 & 2019.2 & 2396.3 & 1660.2 & 2429.6 & 1474.9 & 2396.3 & 1858.8 \\
\hline$\overline{\xi_{F}\left(10^{6} \mathrm{GeV}^{2}\right)}$ & " & "-1.77 & \begin{tabular}{c|}
-2.23 \\
\end{tabular} & $\begin{array}{l}-1.98 \\
\end{array}$ & \begin{tabular}{c|}
-1.21 \\
\end{tabular} & $\overline{-2.32}$ & -1.85 & $\overline{-1.43}$ & $\overline{-1.21}$ & $\overline{-0.47}$ \\
\hline$\overline{\xi_{S}\left(10^{9} \mathrm{GeV}^{3}\right)}$ & $\begin{array}{l}-6.79 \\
\end{array}$ & -3.27 & -6.06 & -6.77 & -5.56 & -3.42 & -6.15 & $\overline{-1.06}$ & $\begin{array}{c}-5.56 \\
\end{array}$ & $\overline{-0.89}$ \\
\hline$m_{\tilde{\chi}_{1}^{0}}(\mathrm{GeV})$ & $\overline{142.2}$ & 181.9 & 112.1 & 146.3 & $\begin{array}{l}33.7 \\
\end{array}$ & 164.5 & 139.5 & 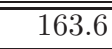 & "33.7 & 94.2 \\
\hline$\overline{m_{h_{1}}(\mathrm{GeV})}$ & $\overline{63.59}$ & 70.59 & $\overline{75.29}$ & 82.24 & 88.07 & 65.93 & 71.32 & 83.77 & 88.07 & 100.47 \\
\hline$m_{h_{2}}(\mathrm{GeV})$ & 122.9 & 122.7 & 122.8 & 123.6 & 126.1 & 127.8 & 126.5 & 124.6 & 126.1 & 125.4 \\
\hline$m_{h_{3}}(\mathrm{GeV})$ & 1858.7 & 1394.5 & 1852.1 & 1861.1 & 2365.0 & 1315.5 & 2078.4 & 950.1 & 2365.0 & 1467.3 \\
\hline$m_{a_{1}}(\mathrm{GeV})$ & 67.8 & 73.4 & 77.8 & 85.3 & 89.0 & 73.9 & 78.2 & 107.1 & 89.0 & 118.9 \\
\hline$m_{a_{2}}(\mathrm{GeV})$ & 2014.3 & 1649.4 & 1995.2 & 2018.0 & 2393.3 & 1659.6 & 2427.6 & 1473.5 & 2393.3 & 1848.9 \\
\hline$m_{h^{ \pm}}(\mathrm{GeV})$ & 112.4 & 114.9 & 121.6 & 124.1 & 103.3 & 102.9 & 101.4 & 124.8 & 103.3 & 120.1 \\
\hline $\mathrm{BR}\left(h_{1} \rightarrow b b\right)$ & 0.902 & 0.910 & 0.909 & 0.901 & 0.828 & 0.910 & 0.909 & 0.906 & 0.828 & 0.907 \\
\hline$\sigma[\mathrm{fb}]$ & 9.783 & 5.627 & 7.535 & 4.815 & 4.628 & 45.209 & 25.561 & 20.205 & 24.371 & 12.403 \\
\hline$\sigma . \mathrm{BR}[\mathrm{fb}]$ & 8.824 & 5.121 & 6.850 & 4.338 & 3.832 & 41.141 & 23.235 & 18.306 & 20.180 & 11.250 \\
\hline
\end{tabular}

Table 3: The selected NMSSM benchmark points obtained using NMSSMTools 5.0.1 [49] to find the $h_{1}$ signal at LHeC. The values displayed are at the electroweak scale. The following parameters are fixed: $M_{3}=1800 \mathrm{GeV}, A_{\tau}=A_{\ell}=1500 \mathrm{GeV}$ and $M_{\tilde{\ell}}=300 \mathrm{GeV}$. Please note that we used $M_{A}$ and $M_{P}$ as inputs - thus our scenario is not the $Z_{3}$-NMSSM, and for that $\xi_{F}$ and $\xi_{S}$ are non-zero and also given in the table. We mention the cross section $\sigma \times \operatorname{BR}\left(h_{1} \rightarrow b \bar{b}\right)$ $($ as $\sigma . B R$ ) at LHeC. Please note that the $e 5$ benchmark for $e+3$-jets and $\nu 4$ benchmark for $\mathbb{F}_{T}+3$-jets is identical.

We have set the renormalization and factorization scales at $\sqrt{\hat{s}}$, the center-of-mass $(\mathrm{CM})$ energy at the parton level, and adopted NN23LO Parton Distribution Functions (PDFs) [68, 69]. including the $b$-flux, with $\alpha_{\mathrm{s}}$ (the strong coupling constant with four-flavor schemes) evaluated consistently at all stages (i.e., convoluting PDFs, hard scattering and decays). Parton shower (both initial and final), hadronization, heavy hadron decays, etc. have been dealt with by PYTHIA v.6.428 [70].

We consider all the light-flavor quarks, $b$-quark and gluon in the proton flux. The flavormixing, wherever appropriate, is also considered for the allowed diagrams. Following this, it was realized that the signal processes have unique kinematic profiles and we will discuss it below.

The cross section of the scattering $f a \rightarrow f^{\prime} X$ via the gauge boson $(V)$ exchange can be expressed as

$$
\sigma\left(f a \rightarrow f^{\prime} X\right) \approx \int d x d p_{T}^{2} P_{V / f}\left(x, p_{T}^{2}\right) \sigma(V a \rightarrow X)
$$

where the fermion $f$ with a c.m. energy $E$ is radiating a gauge boson $V\left(s \gg M_{V}^{2}\right)$, 
$\sigma(V a \rightarrow X)$ is the cross-section of the $V a \rightarrow X$ scattering and $P_{V / f}$ can be viewed as the probability distribution for a weak boson $V$ of energy $x E$ and transverse momentum $p_{T}$. The dominant kinematical feature is a nearly collinear radiation of $V$ off $f$, termed as "Effective $W$-Approximation" [71]. The probability distributions of the weak bosons with different polarizations can be approximated by (in the limit of $s \gg M_{V}^{2}$ )

$$
\begin{aligned}
& P_{V / f}^{T}\left(x, p_{T}^{2}\right)=\frac{g_{V}^{2}+g_{A}^{2}}{8 \pi^{2}} \frac{1+(1-x)^{2}}{x} \frac{p_{T}^{2}}{\left(p_{T}^{2}+(1-x) M_{V}^{2}\right)^{2}} \\
& P_{V / f}^{L}\left(x, p_{T}^{2}\right)=\frac{g_{V}^{2}+g_{A}^{2}}{4 \pi^{2}} \frac{1-x}{x} \frac{(1-x) M_{V}^{2}}{\left(p_{T}^{2}+(1-x) M_{V}^{2}\right)^{2}}
\end{aligned}
$$

where $g_{V}\left(g_{A}\right)$ is the vector (axial) vector couplings of fermion-gauge boson vertices.

From these equations we can understand that the final state quark $f^{\prime}$ typically has transverse momentum, $p_{T} \sim \sqrt{1-x} M_{V} \leq M_{V}$, i.e, less than the mass of the vector boson. Secondly, due to the $1 / x$ behavior for the gauge boson distribution, the out-going parton energy $(1-x) E$ tends to be high and leads to very high energetic forward jet, with small angle (i.e., high forward rapidity) with respect to the beam direction. Finally, at high $p_{T}$, the probabilities of the gauge bosons can be approximated as: $P_{V / f}^{T} \sim 1 / p_{T}^{2}$ and $P_{V / f}^{L} \sim 1 / p_{T}^{4}$. At high $p_{T}$ the longitudinally polarized gauge bosons is relatively suppressed as compared to the transversely polarized one. In particular, the first two criterions serve as a guidance in our event selection for exploiting the kinematical features. Also, in both signals under consideration, the final state forward jet could be also a $b$-jet. However, as it is mostly in the forward region, with the tighter constraints of the rapidity of $b$-taggable jet, it hardly qualifies as $b$-tagged.

\subsection{Backgrounds}

There are mainly two groups of SM backgrounds in our Higgs signals. The charged-current backgrounds consisting of $\nu t \bar{b}, \nu b \bar{b} j, \nu b 2 j, \nu 3 j$, and the neutral-production ones identified as $e^{-} b \bar{b} j, e^{-} t \bar{t}, e^{-} b j j$ and $e^{-} j j j$. In all of these backgrounds the charge-conjugated processes are naturally implied.

For estimating the cross sections of these SM backgrounds, we used the same set of preselections, identical conventions and parameter sets as for the signal. The expected number of the background events for $100 \mathrm{fb}^{-1}$ of integrated luminosity are given in the second column of Tables 4 and 6 .

\subsection{Signal-to-Background analysis}

We generated the SM backgrounds at the parton level using MadGraph v 2.4.3 63] and then fed them to PYTHIA v.6.428 [70] for parton showering (both initial and final), hadronization, heavy hadron decays etc. The initial state radiation (ISR) will reduce the total center-of-mass energy of the collision, however at the LHeC with the main dynamics along the t-channel, the center-of-mass energy loss due to ISR has less impact. The top-quark and $W$-boson were allowed to decay freely within PYTHIA program. The four-momentum of the jets are different as compared to the parton level quark due to the final state radiation (FSR) and in our analysis we considered the Gaussian type of smearing effects. 
The LHeC detectors and their parameters considerations follow one of our recent analysis [36]. However to be complete, let us describe it here briefly.

We have considered the experimental resolutions of the jet angles and energy using the toy calorimeter PYCELL, in accordance with the LHeC detector parameters, given in PYTHIA. As the invariant mass has been used to isolate the Higgs signal - this has some non-trivial effect. We considered LHC type of calorimeter for the LHeC. To be explicit, we set a somewhat symmetric detector coverage, however in reality the electromagnetic and the hadronic calorimeter at LHeC, unlike ATLAS and CMS, are not exactly symmetric.

Since we are not doing detector simulation and also not considering the cracks in the detectors, we applied symmetric large rapidity coverage for jets and leptons 7 . We expect that these assumptions hardly alter our numerical findings. The detector parameters in PYCELL are set according to the LHeC detector [66]. Specifically, we assume large calorimeter coverage $|\eta|<5.5$, with segmentation (the number of division in $\eta$ and $\phi$ are 320 and 200, respectively) $\Delta \eta \times \Delta \phi=0.0359 \times 0.0314$. Furthermore, we have used Gaussian energy resolution [64] for electron and jets (labeled as $j$ ), with

$$
\frac{\Delta E}{E}=\frac{a}{\sqrt{E}} \oplus b
$$

where $a=0.32, b=0.086$ for jets and $a=0.085, b=0.003$ for leptons and $\oplus$ means addition in quadrature.

We have used a cone algorithm for the jet-finding, with jet radius $\Delta R(j)=\sqrt{\Delta \eta^{2}+\Delta \phi^{2}}=$ 0.5. Calorimeter cells with $E_{T, \text { min }}^{\text {cell }} \geq 5.0 \mathrm{GeV}$ are considered to be potential candidates for jet initiators. All cells with $E_{T, \text { min }}^{\text {cell }} \geq 1.0 \mathrm{GeV}$ were treated as part of the would-be jet. A jet is required to have minimum summed energy $E_{T, \text { min }}^{j} \geq 15 \mathrm{GeV}$ and the jets are ordered in $E_{T}$. The leptons $(\ell=e$ only $)$ are selected if they satisfy the requirements: $E_{T}^{\ell} \geq 15 \mathrm{GeV}$ and $\left|\eta^{\ell}\right| \leq 3.0$. In our jet finding algorithm we include leptons as parts of jets. Finally we separate them, putting some isolation criterion as follows: if we find a jet near a lepton, with $\Delta R(j-\ell) \leq 0.5$ and $0.8 \leq E_{T}^{j} / E_{T}^{\ell} \leq 1.2$, i.e. if the jet $E_{T}$ is nearly identical to that of this lepton, the jet is removed from the list of jets and treated as a lepton. However, if we find a jet within $\Delta R(j-\ell) \leq 0.5$ of a lepton, whose $E_{T}$ differs significantly from that of the lepton, the lepton is removed from the list of leptons. This isolation criterion mostly removes leptons from $b$ or $c$ decays. We reconstructed the missing (transverse) energy $\left(\mathbb{F}_{T}\right)$ from all observed particles and for the charge current the signal is shown in right panel of Fig. 9, We have also calculated the same from the energy deposition in the calorimeter cells and found consistency between these two methods. Only jets with $\left|\eta^{j}\right|<2.5$ and $E_{T}^{j} \geq 15 \mathrm{GeV}$ "matched" with a $b$-flavored hadron ( $B$-hadron), i.e. with $\Delta R(j, B$ - hadron $)<0.2$ is considered to be "taggable". We assume that these jets are actually tagged with probability $\epsilon_{b}=0.50$. We also adopted mis-tagging of non- $b$ jets as $b$-jets and treated $c$-jets differently from the gluon and light-flavor jets. A jet with $\left|\eta^{j}\right| \leq 2.5$ and $E_{T}^{j} \geq 15 \mathrm{GeV}$ matched with a $c$-flavored hadron ( $C$-hadron, e.g., a $D$-meson or $\Lambda_{c}$-baryon), i.e., with $\Delta R(j, C$ - hadron $)<0.2$, is again considered to be taggable, with (mis-)tagging probability $\epsilon_{c}=0.10$. Jets that are associated with a $\tau$-lepton, with $\Delta R(j, \tau) \leq 0.2$, and all jets with $\left|\eta^{j}\right|>2.5$, are taken to have vanishing tagging probability. All other jets with $E_{T}^{j} \geq 15 \mathrm{GeV}$ and $\left|\eta^{j}\right| \leq 2.5$ are assumed to be (mis-

\footnotetext{
${ }^{7}$ Here the lepton means only electron unless mentioned otherwise.
} 
)tagged with probability $\epsilon_{u, d, s, g}=0.01$, following [34]. The overall analysis strategy has been adopted from [72, 73] and [74].
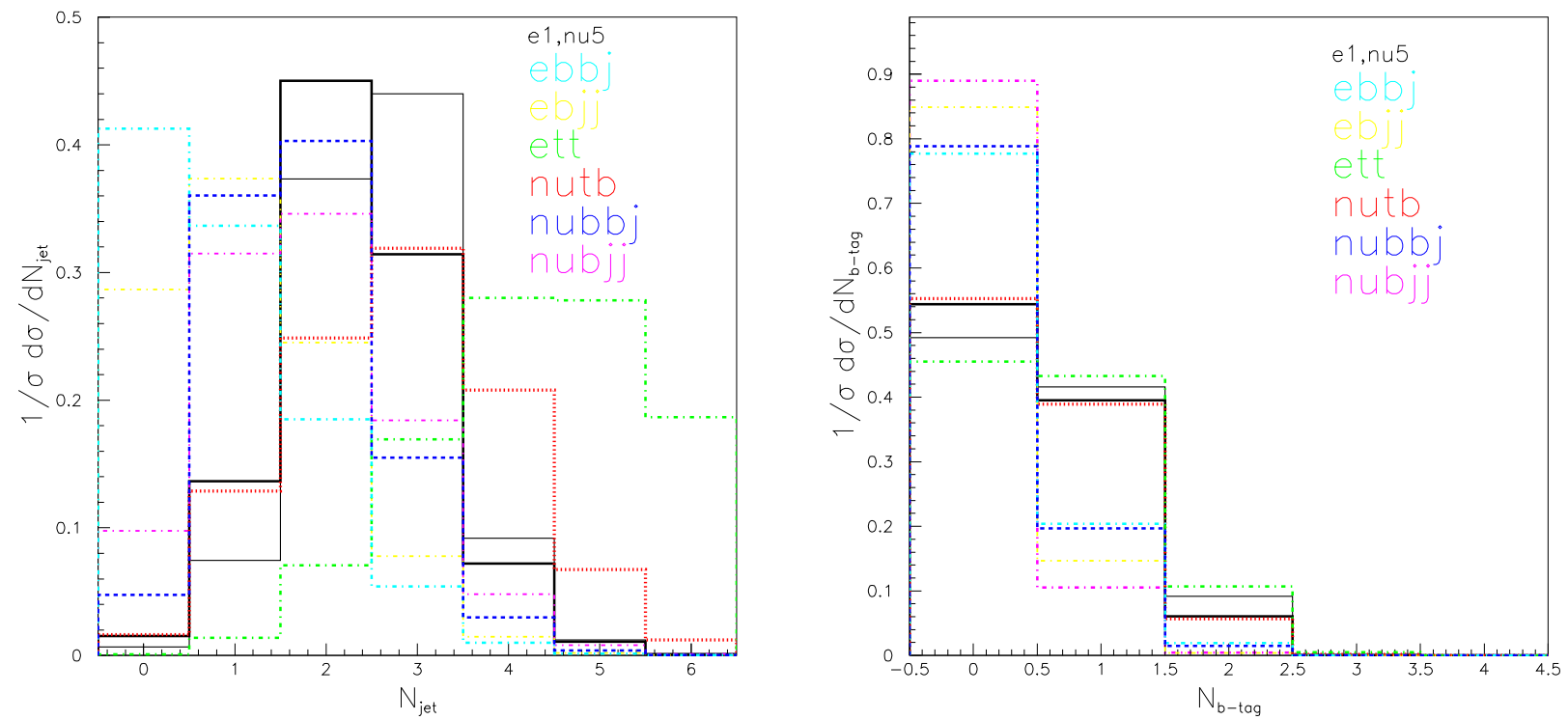

Figure 8: Left-panel: Number of jet distribution for the $e 1$ benchmark ( $\nu 5$ benchmark) in the $e+3$-jets $\left(\mathscr{F}_{T}+3\right.$-jets) channel using the thick (thin) black lines. Right-panel: The number of $b$-tagged jet distribution is shown. Both the distributions depends mainly upon the masses of the Higgs boson in two signal channels, i.e., the more massive the Higgs, the more the number of jets peaks towards higher values. For all other signal benchmarks, the distributions follow a similar pattern and can be understood from the numerics in the corresponding columns in Tables 4 and 6.

\subsection{1 $e+3$-jet: electron channel}

In this subsection we will analyze the neutral current signal electron channel, i.e., $e+3$-jet and apply different kinematical cuts to isolate the signal from the backgrounds.

- a: We first selected events containing at least three jets, i.e., $N_{\text {jet }} \gtrsim 3$, with $P t_{j}>15.0$ and $\eta_{j}<5.5$. The distribution of the number of jet $\left(N_{\text {jet }}\right)$ is shown in the left panel of Fig. 8. The efficiency of having $N_{\text {jet }} \gtrsim 3$, are approximately, 40.0\%, 42.8\%, 45.3\%, $45.6 \%$ and $51.7 \% 8$ for the signal benchmarks of $e 1$ to $e 5$, respectively. Since the two central jets originate directly from the Higgs boson itself, heavier masses lead to higher efficiencies. The jet selection criterions are the same for all the signals and corresponding backgrounds. Thus the jet efficiencies are identical in all backgrounds. Among all the backgrounds, et $\bar{t}$ leads to a total of six-jets when both the top-quarks decay hadronically - here the jet efficiency is maximal, about $92.2 \%$. The immediate next high efficiency is

\footnotetext{
${ }^{8}$ The efficiencies quoted here are with respect to the previous set of selections.
} 

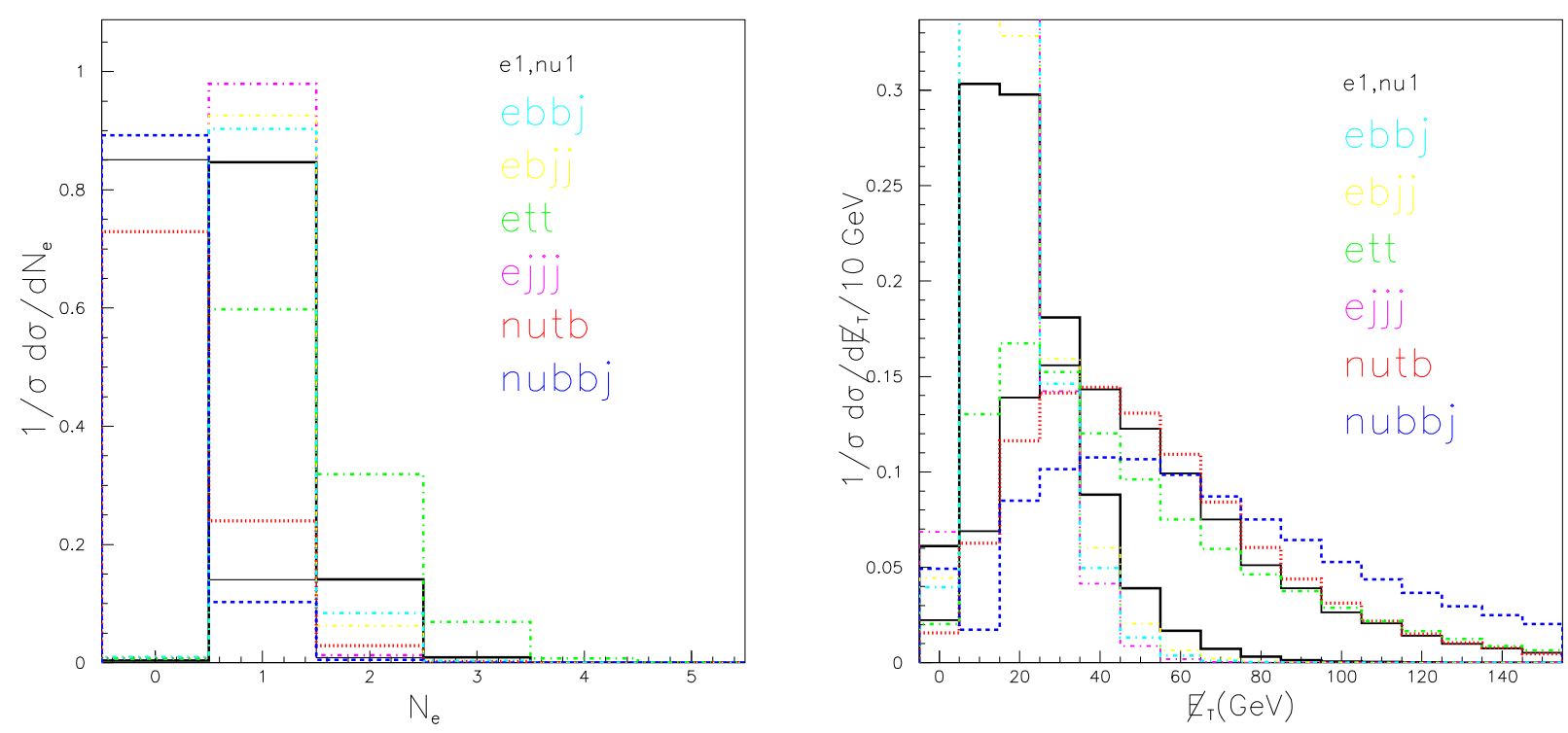

Figure 9: Number of electron $\left(N_{e}\right)$ distribution for $e 1$ benchmark ( $\nu 1$ benchmark) using thick (thin) black line in the left panel. The distribution of missing energy $\left(\mathbb{F}_{T}\right)$ for $e 1(\nu 1)$ benchmark using thick (thin) black line in the right panel.

from $\nu t b$ - where the maximal number of jets is four. This leads to an efficiency around $60.7 \%$. The jet-efficiency for the ebbj, ebjj and ejjj are in between $7.0-10.0 \%$ whereas for the $\nu b b j, \nu b j j$ and $\nu j j j$ channels they range from 18.0 to $24.0 \%$. In the neutrino cases, first of all there is no strict selection of neutrinos momentum unlike minimum transverse momentum requirements of the lepton, i.e., $e$ in this channel. Moreover the lepton-jet isolation criterion reduces the number of jets in the backgrounds with explicit lepton. This leads to the lower efficiency.

- b: We required one lepton $(e)$ with $p_{T}>15 \mathrm{GeV}$ and $\eta<3.0$ as our signal is generated from the neutral current interaction. Since the lepton is originating from the $e$-beam, we required somewhat larger rapidity. The distribution of number of leptons is shown in the left panel of Fig. 9. The lepton efficiency for all the signal benchmarks is approximately 94.0\% and for the benchmarks where the Higgs mass is smaller, this lepton efficiency is larger as more collison energy is transferred to the lepton - however small it is, it does get reflected in the corresponding efficiencies. Among the backgrounds, the larger efficiency is for et $\bar{t}$ with approximately $86.5 \%$ - as one top quark is allowed to decay leptonically whereas the other top quark must decay hadronically satisfying the three jet criterion. The efficiencies for ebbj, ebjj and ejjj are between $76.9 \%-79.7 \%$. The efficiency of $\nu t b$ is approximately $8.0 \%$. This is close to the electron channel branching fraction, i.e., $10 \%$. There are also secondary sources of electrons, like semi-leptonic $b$-decays or meson decays. Taking this into account, the transverse momentum and rapidity criterion reduces the efficiency to approximately $2 \%$. The efficiency for $\nu b b j$ is approximately $2.8 \%$ where the source of the lepton is only from the semi-leptonic b-decay satisfying the isolation 
criterion or from secondary sources like meson or photon. The efficiency for $\nu b j j$ being $1.3 \%$ is just the half of $\nu b b j$ as it is clear from the presence of b-quark in these two cases. In case of $\nu j j j$, the lepton would only be coming from the secondary sources (meson or photo) during fragmentation and hadronization and the efficiency turns out to be approximately $0.002 \%$.

- c: We demanded at least two $b$-tagged jets with the inclusion of proper mis-tagging. The distributions of the number of $b$-tagged jets $\left(N_{b-t a g}\right)$ are shown in the right panel of Fig. 8. The efficiencies are approximately, $13.7 \%, 13.8 \%, 14.4 \%, 14.1 \%$, and $14.6 \%$ for the $e 1$ to $e 5$ benchmark points, respectively. In fact, all our signal benchmarks contain at least two $b$-quark. Since we considered $\epsilon_{b}=0.50$, an approximately $10 \%$ lowering for the double $b$-tag is quite realistic due to the fact that not all $b$-quarks in the signal are eligible for the $b$-taggable criterion adopted in our analysis (another possibility is having a $c$-quark with $\epsilon_{c}=0.10$ and a light flavored quark with $\left.\epsilon_{q}=0.01\right)$.

Among the backgrounds, the irreducible one $e b b j$ has an efficiency of approximately $11.5 \%$, roughly $2 \%$ less than the signal and this is mainly due to the rapidity acceptance of the taggable $b$-jet. Unlike the signal, the rapidity distribution of the two $b$-taggable jet for this background are not very central (i.e., we imposed the $\eta<2.5$ a for taggable $b$-jet). For $e t b$ and $\nu t b$ the efficiencies are approximately $11.6 \%$ and $10.0 \%$, respectively whereas $\nu b b j$ and $\nu b j j$ have efficiencies of $8.2 \%$ and $2.4 \%$. This $b$-tagged ratios with neutrino follow very closed the corresponding number efficiencies of $e b b j$ and $e b j j$. The efficiency of ejjj is approximately 0.002 where low-flavor mis-tagging efficiencies with $\epsilon_{c}=0.10$ and $\epsilon_{q}=0.01$ $(q=u, d, s, g)$ have been taken care of. We would expect similar $b$-tagged efficiencies (i.e, 0.002) for $\nu j j j$. However, as the lepton selection criterion (b) above severely reduced the number of events - the survived events hardly pass this $b$-tag criterion and $\nu j j j$ goes to zero-level.

- d: In the central region, defined above in the selection (c), having the number of btagged jets greater than or equal to two (i.e., $N_{b} \gtrsim 2$ with mis-tagging), we reconstruct all possible combination of di-jet invariant masses, i.e., $M_{b b}$. Out of all possible combinations, we have chosen the best combination for which the absolute difference, $\left|M_{b b}-M_{h_{1}}\right|$ is minimum. We identified this as the correct di- $b$-jet candidates for the Higgs boson. The distribution of $M_{b b}$ is shown in the left panel of Fig 10 for $e 1, e 3$ and $e 5$ benchmark points. We have not shown explicitly the mass peaks of $e 2$ and $e 4$ benchmark points, since it lies between the displayed peaks as the Higgs boson mass lies between the respective benchmarks (see Table 3). The peaks of all the signal benchmarks always show up to the left side of the actual masses due to jet energy smearing. Moreover, the shift depends on the jet-cone size under considerations - the larger the cone size the more the peak moves to the right. The price is having a relatively less $N_{j e t}$ efficiency in (a). The $M_{b b}$ distributions in Fig.10 show a rapid fall on the higher side. Hence we have selected events with some asymmetric mass windows: $M_{h_{1}}-15.0 \mathrm{GeV}<M_{b b}<M_{h_{1}}+5 \mathrm{GeV} 10$.

\footnotetext{
${ }^{9}$ Please note that we have not considered here the lepton mis-tagging efficiency from the jets. This is approximately $0.001 \%$ and having the three (or more) jets explicitly after considering the ISR and FSR, this efficiencies are somewhat consistent with the mis-tagging numbers with proper combinatorics.

10 Please note that the $M_{b b}$ distributions are shown without imposing the mass window selection. The
} 

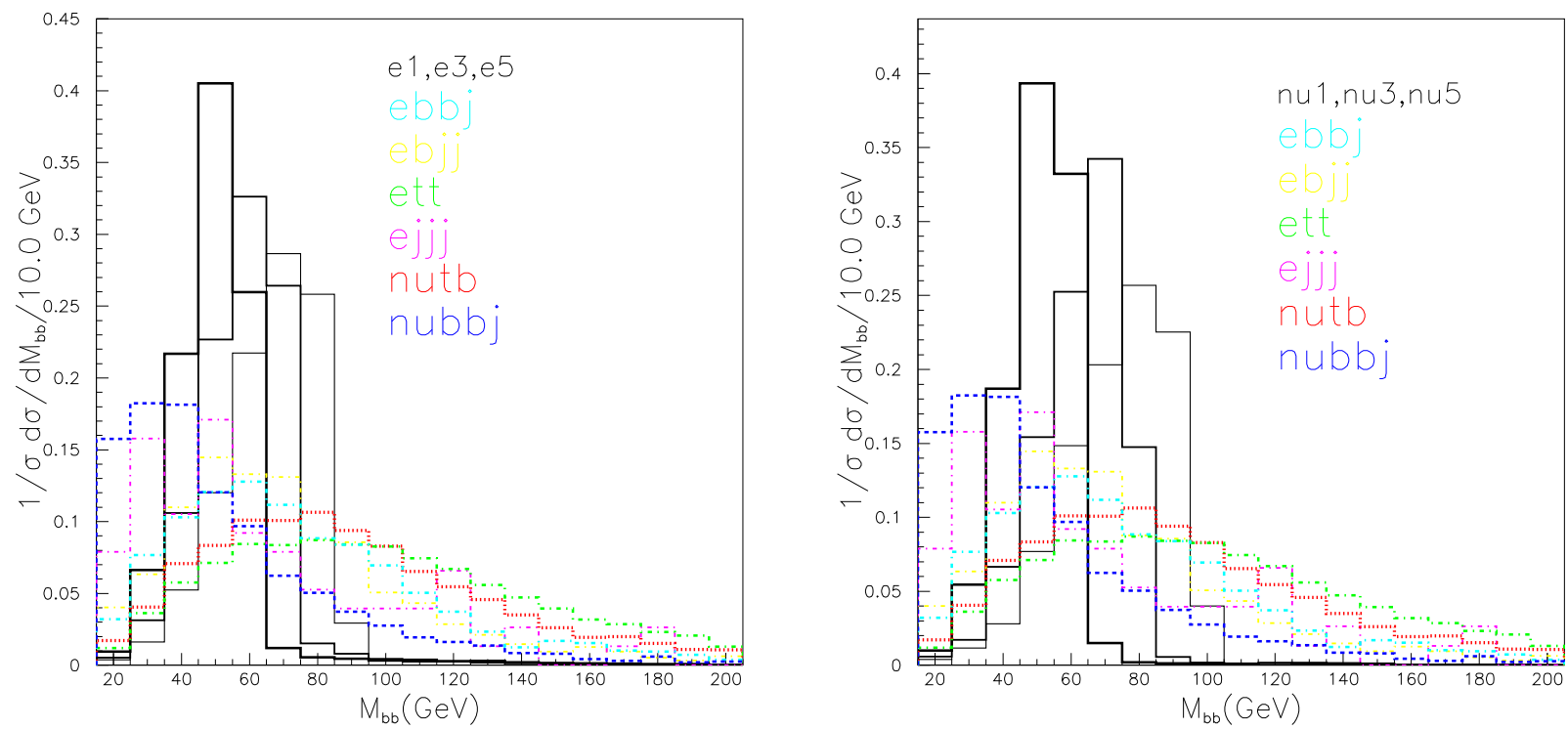

Figure 10: Left-panel: The di-b-tagged invariant masses, $M_{b b}$, distribution for the $e+3$-jets channel, for $e 1, e 3$ and $e 5$ benchmark points from left to right (thick to thinner) respectively. Right-panel: Similar for the $\mathbb{E}_{T}+3$-jets channel, with the mass peaks for $\nu 1, \nu 3$, and $\nu 5$ from left to right (thick to thinner) respectively. See the Table 3 for the corresponding Higgs boson masses.

For the signal the efficiencies are approximately, $54.0 \%, 47.7 \%, 43.3 \%, 38.7 \%$, and $35.0 \%$ for $e 1$ to $e 5$ benchmark points, respectively. The reconstructed Higgs boson mass tends to lie in the lower regions which depends mainly on the jet-cone size and the showering effects. Thus, the lower the benchmark Higgs boson mass, the larger is the efficiency as can be seen from the second benchmark point.

The SM $e b b j$ processes have a $Z$-boson exchange resonant diagram with $Z \rightarrow b \bar{b}$. This leads to the appearances of the mass peaks around $60 \mathrm{GeV}$ (approximately $30 \mathrm{GeV}$ less than the $M_{Z}$ due to jet energy smearing). The $\nu$ bjj mass peaks are somewhat similar to the ebjj, as this has a $W$ and/or $Z$-boson exchange resonant diagrams. As the Higgs boson mass is very close to the $W$-boson mass at least in the $e 1$ benchmark, the efficiencies are $32.5 \%$ and $25.4 \%$ for $\nu b j j$ and $e b j j$, respectively.

In the cases of ett and $\nu t b$, the pure di-b-tagged jet is uncorrelated and as a result the mass distributions are flat. However, it is not always the case that the primary hard $b$-jet or the secondary $b$-jet from the top quark decay are tagged. In the cases, of the hadronic top decay, i.e., W-hadronic decay, both can be mis-tagged and those combinations generally show the mass peaks around $80-90 \mathrm{GeV}$. The efficiencies of this selections are approximately around $15.3 \%$ and $18.1 \%$ for ett and $\nu t b$, respectively.

numbers start to differ from this column onwards as the Higgs mass window selection depends upon the Higgs boson masses of the corresponding benchmarks. 
The efficiency for ejjj is approximately $18.2 \%$ and the mass distribution has a peak in the lower side. Due to this peak shift in the lower side, among all the benchmarks the ejjj events survive better than of the benchmarks with minimum Higgs boson mass.
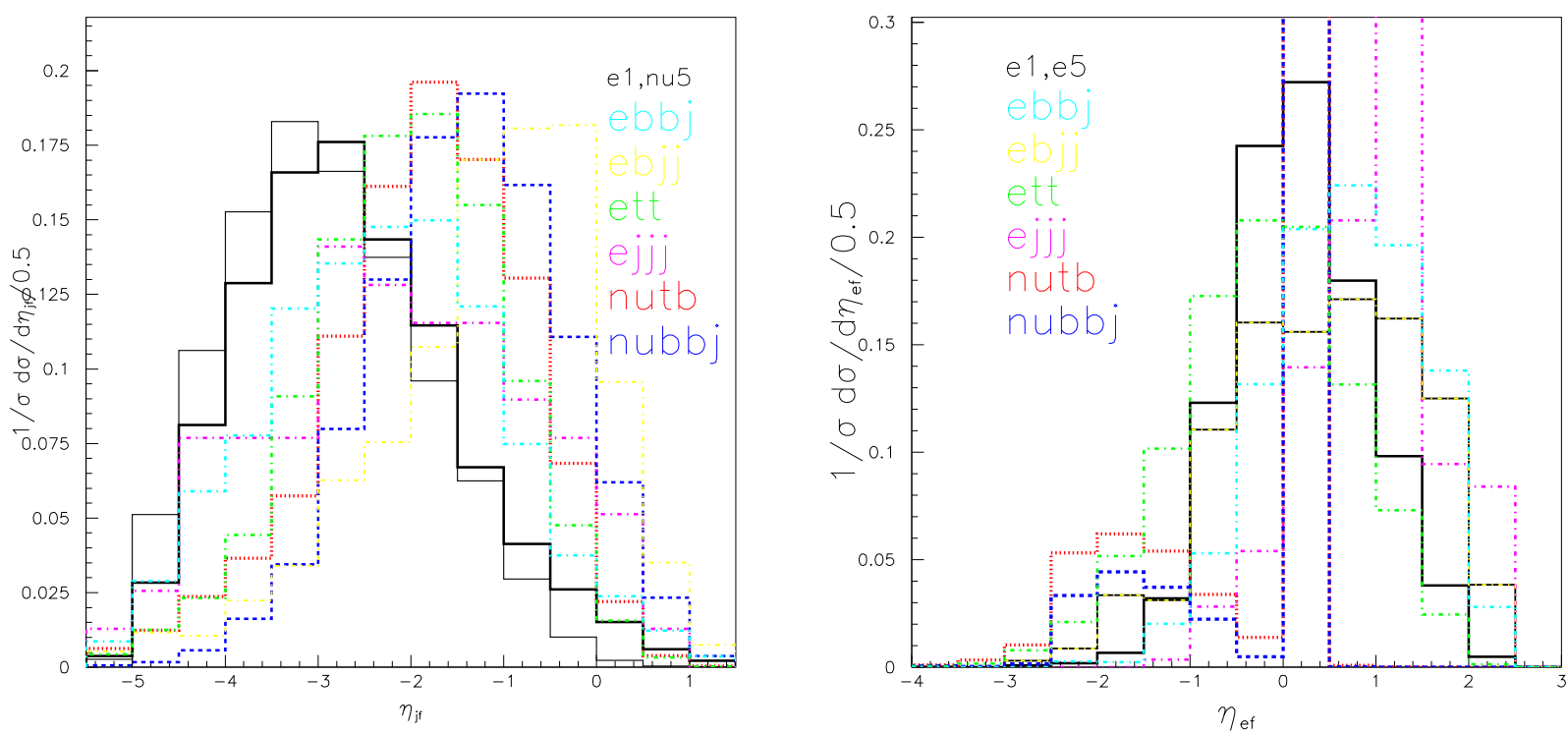

Figure 11: Left-panel: The rapidity of the forward jet $\left(\eta_{j f}\right)$ for $e 1$ and $\nu 5$ signal benchmark points. The distributions for other benchmarks lie between this distribution profiles. Rightpanel: The rapidity of the forward lepton $\left(\eta_{e f}\right)$ for $e 1$ and $e 5$ benchmark points (somewhat similar for other benchmarks).

- e: We demanded that the remaining leading jet in the events should have $P t_{j}>15.0 \mathrm{GeV}$ with $-5.5<\eta<-0.5$ (the values are chosen by inspecting the distribution shown in the left panel of Fig. 11 with thick black line for $e 1$ benchmark and termed as the forward jet $\left(j_{f}\right.$ or in the histogram as $\left.j f\right)$ ). The forward jet (the transverse momentum of the forward jet is shown in the left-panel of Fig.12) lies very close to the direction of the incoming proton - however it also depends upon the Higgs mass for the given benchmarks. The more massive the Higgs boson is, the smaller the energy which remains for the forward jet as it lies close to the proton beam (larger rapidity). Vice versa, the less massive the Higgs, the larger the rapidity of the forward jet. With the exception of the $e 2$ benchmark, the survival probability under this selection is larger with heavy Higgs boson masses. The backgrounds like $\nu t b$ and ett have generally a large number of jets (shown in the left panel of Fig. 8). Thus one out of many jets would likely pass this selection criterion and hence the efficiencies are as large as the signal efficiency. The backgrounds ebjj and $\nu b j j$ have somewhat similar efficiencies and are reduced maximally by this selection.

After applying this selection, the dominant remaining backgrounds are from the irreducible $e b b j, e b j j, \nu t b$ and a part from ejjj as the latter process has a big cross-section to begin with. 
- f: We demand the magnitude of product of lepton and jet rapidity to be negative, i.e., $\eta_{j} . \eta_{l}<0$. This is to say that they lie in the opposite hemisphere. For this selection, the efficiencies for the $e 1$ to $e 5$ signal benchmarks, vary, as $53.9 \%, 55.2 \%, 55.9 \%, 57.8 \%$ and $55.8 \%$ respectively. This selection reduces the high multiplicities backgrounds severely. For example, $\nu t b$ (ett) survived by approximately 1.5\% (39.5\%).

- g: Like the forward jet cut mentioned above in the item (e), we assume the rapidity of the lepton to be in the forward region since the lepton in the signal source is directly from the $e$-beam 11. The transverse momentum of the forward lepton is shown in the rightpanel of Fig.12. By inspecting the rapidity distribution of this lepton shown in the right panel of Fig 11, we selected events with lepton rapidity within the range $-2.5<\eta<2.0$. The lepton satisfying this criterion is called forward lepton $\left(e_{f}\right)$. The signal events which survived this criterion are approximately $94.0 \%$ while for $\nu t b$ it reduces to approximately $70 \%-85 \%$ as the source of the lepton in this case is the top quark decay and there is no guarantee that the lepton should be in the forward direction with the imposed criterion. The background ett would be maximally three leptons and it is likely that one out of three leptons will pass the forward rapidity criterion $-2.5<\eta<2.0$. Thus one would expect large survival probability which indeed is approximately $80 \%$. In Table 4 we tabulated the events after imposing the rapidity gap between the forward lepton and forward jet, i.e., $\Delta \eta_{j f-e f}$. We have shown the distribution of rapidity of lepton in the right-panel of Fig,11 and the rapidity differences with jet in the left-panel of Fig.13. We demanded -5.5 $<\Delta \eta_{j f-e f}<0.5$ and due to that the signal benchmarks reduced by approximately $2.0-$ 3.0\%. All the SM backgrounds remain same except the ebjj changes from $93.3 \%, 94.9 \%$, $95.7 \%, 99.2 \%$ and $96.1 \%$ for $e 1$ to $e 5$ signal benchmarks, respectively.

- h: For the di- $b$-jet for which $M_{h_{1}}=m_{b b}$ as in the selection (d) above and with the forward tagged jet $\left(j_{f}\right)$, we reconstructed three-jet invariant mass, $m_{h_{1} j_{f}}=m_{b b j_{f}}$. This essentially reflects the overall energy scale of the hard scattering. The distributions are shown in the right-panel of Fig. 13. We impose the condition $m_{h_{1} j_{f}}>190 \mathrm{GeV}$. With this selection the signal events for all the benchmarks remain approximately $50.2-54.5 \%$. The most dominant irreducible background ebbj remains approximately $14.0 \%-24.0 \%$ whereas ebjj remains approximately $7.0 \%$ or less. The backgrounds ett remains approximately $8.0 \%$ or less. The $\nu t b$ becomes zero.

At this stage the most dominant backgrounds which remain are: $e b b j$, ebjj (at the level of signal events) and ejjj.

- i: We devised another set of selection based on the sum of the transverse momentum of all jets present in the events, $H_{T}=\sum\left|P t_{j}\right|$. The distribution is shown in the left panel of Fig. 14. The signal shows the peak around $100 \mathrm{GeV}$. The $\nu t b$ shows a peak around 125 $\mathrm{GeV}$ whereas ett displays it around $250 \mathrm{GeV}$ - the higher value reflects the presence of more number of jets. We demanded a selection of $H_{T}>100 \mathrm{GeV}$. The number of signal events for all the benchmarks remains approximately 95.0\% - 98.0\% (for heavy Higgs

\footnotetext{
${ }^{11}$ Please note that the lepton(i.e, $\left.e\right)$ and the forward jet $\left(j_{f}\right)$ are likely to be in the opposite hemisphere, i.e., if the jet is in the forward region then the lepton will be in the backward region or vice-versa. This is also reflected in the rapidity distributions in the left and right panel of Fig[11.
} 

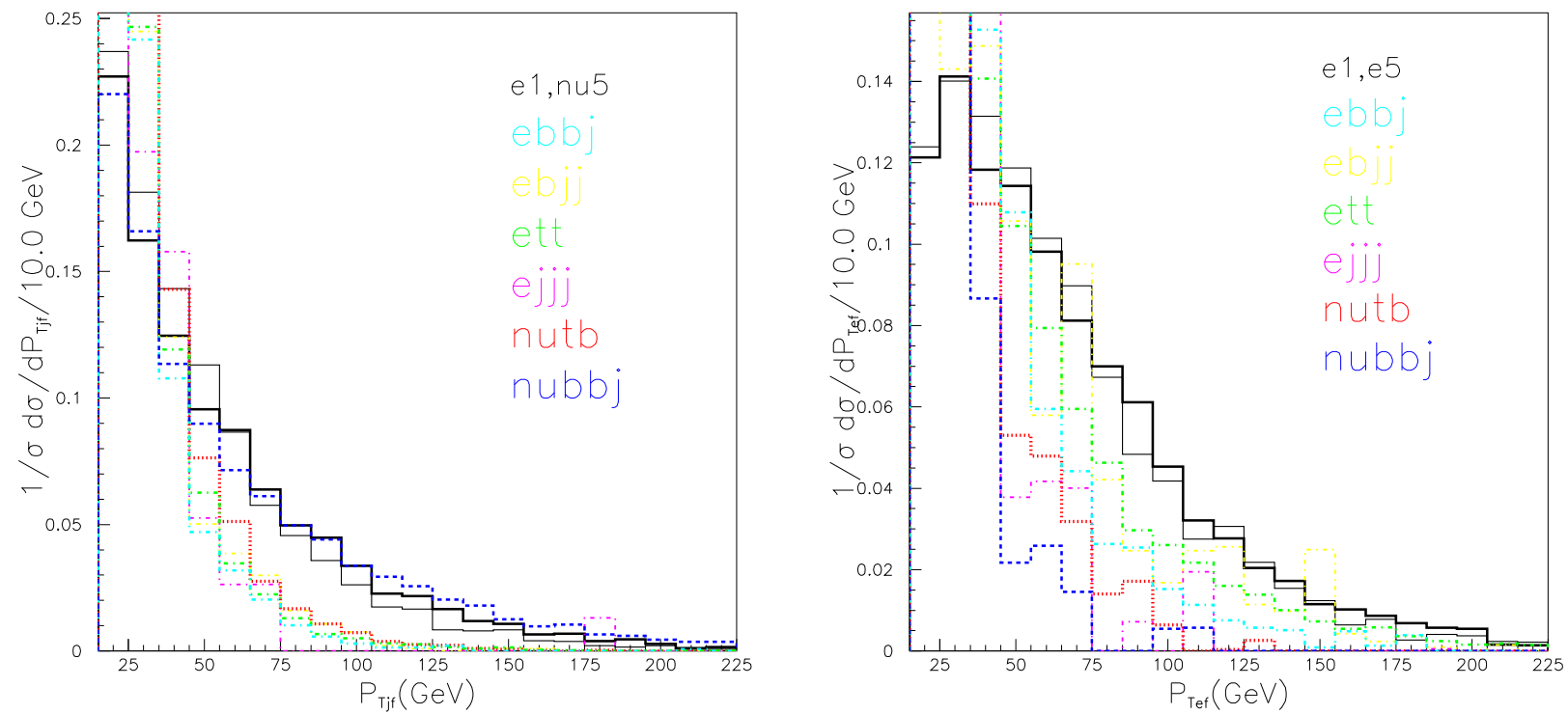

Figure 12: Left-panel: The transverse momentum $\left(P_{T}\right)$ of forward jet $\left(P_{T j f}\right)$ for e1 and $\nu 5$ signal benchmarks. Right-panel: The same for the forward lepton $\left(P_{T e f}\right)$ only in the electron channel for $e 1$ and $e 5$ signal benchmarks..

boson masses the survival events probablities are more). We see that this selection is not reducing much of ett, at most $2.0 \%$. However this background is not big at this stage. The ebjj contribution is also not much, it survived by at most $75.0 \%$ for $e 5$ benchmark. With this selection $e b b j$ is reduced to approximately $52.2 \%, 55.6 \%, 64.0 \%, 65.5 \%$ and $73.9 \%$ for $e 1$ to $e 5$ benchmark points respectively. In spite of large large reduction, ebbj is the only dominant contribution at this stage.

- $\mathbf{j}$ : Finally, to suppress the ebbj further, we devise a new kinematical variable by adding the vector component of three momentum of all the jets present in the event. This is defined as: $\vec{H}_{T}=\sum \vec{P} t_{j}$. The distribution is shown in the right panel of Fig. 14 and it is evident that here the peaks are at a lower value of $\left|\vec{H}_{T}\right|$ as compared to the left panel of the same figure ( $H_{T}$ is defined in the selection (i) above). In the events having more jets, the jets are naturally distributed symmetrically in the $\eta-\phi$ plane. This regular arrangements of jets three momentum tend to cancel each other which leads to a lower magnitude of $\vec{H}_{T}$. For example, for ett, $\nu t b$ the peaks are around $40 \mathrm{GeV}$, while for the $e b b j$, ebjj, and ejjj around $25 \mathrm{GeV}$ or less. By demanding the magnitude of $\left|\vec{H}_{T}\right|>50$ $\mathrm{GeV}$, the signals are reduced by approximately $30.0 \%-35.0 \%$. The background $e b b j$ is reduced severely by approximately $85 \%-95 \%$.

After applying the cumulative selection from (a)-(j), we estimated the total SM background events to be found in the final column of Table 4. The significance for $100 \mathrm{fb}^{-1}$ integrated luminosity is approximately $0.40-0.12 \sigma$. This is not good enough to ensure the finding of a Higgs boson. However, at the end of LHeC data accumulation with approximately $1000 \mathrm{fb}^{-1}$, 

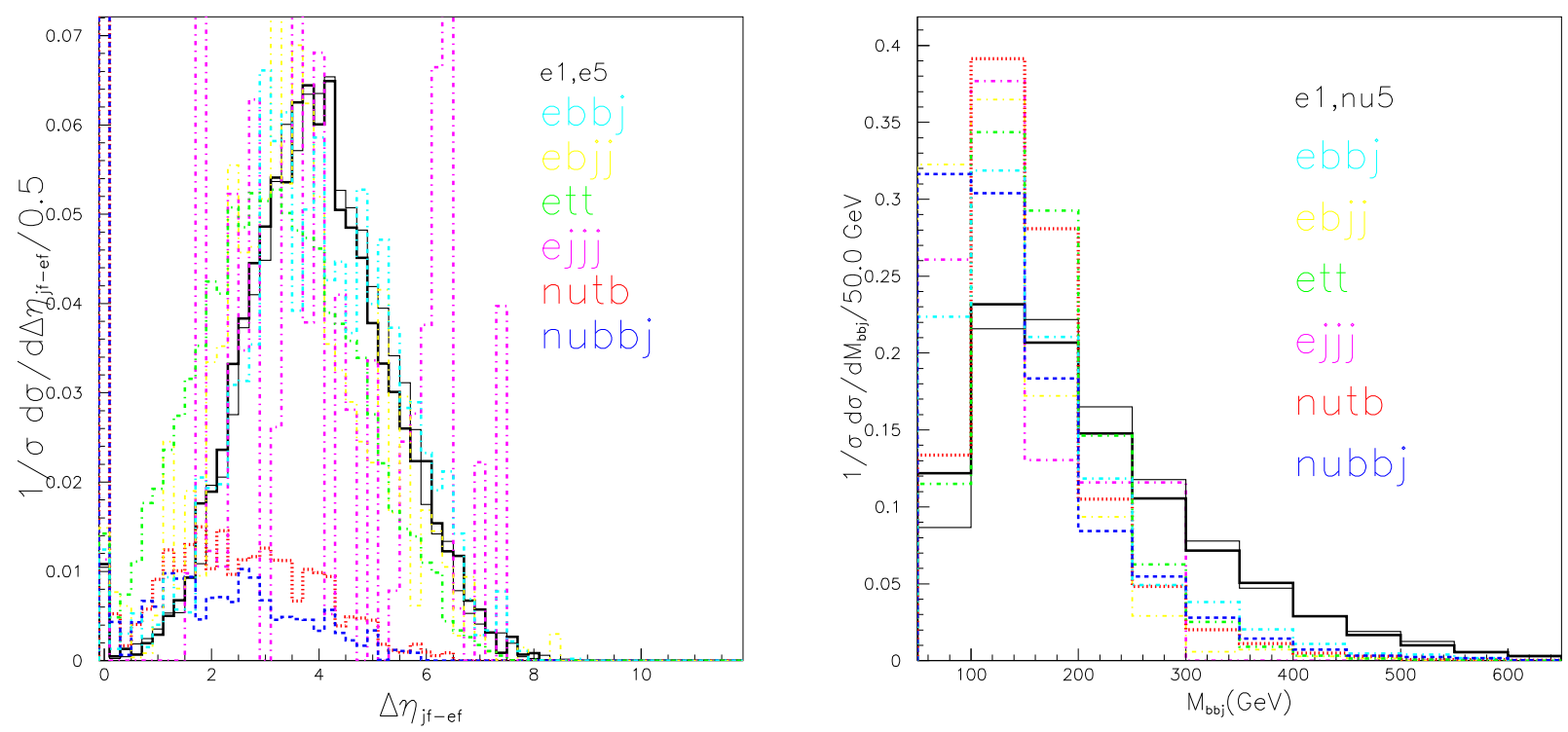

Figure 13: Left-panel: The rapidity differences between forward jet $\left(j_{f}=j f\right)$ and forward lepton $\left(e_{f}=e f\right)$, i.e., $\Delta \eta_{j f--e f}$ only for $e 1$ and $e 5$ signal benchmarks in electron channel. The signal shows large rapidity gap. Right-panel: The invariant masses of the Higgs candidate jets, $M_{b b}$ shown in Fig,10, together with the forward jet, i.e., $M_{b b j_{f}}=m_{h_{1} j_{f}}$ for both the signal channels for $e 1$ benchmark ( $\nu 5$ benchmark) using thick(thin) black lines. The signal distributions in both channels do not differ as the electron and $\mathbb{F}_{T}$ does not have a direct big role to reconstruct the three-jet invariant mass. The distributions for other signal benchmarks in both the channels are somewhat identical.

the significances (quoted in the parenthesis) improve to become $1.3-0.4 \sigma$. Notice that the significances do not scale accordingly with the Higgs masses. For example, the mass of the Higgs boson in $e 2$ benchmark is less than the $e 3$ benchmark, thus one would expect the larger cross-section in $e 2$ benchmark. This is mainly because of the parameter spaces dependent couplings of the $Z Z h_{1}$ in the gauge boson fusion type of production. For the $e 2$ benchmark, the values of $Z Z h_{1}$ coupling is less with respect to $e 3$ benchmark.

It is somewhat clear from Table 4 that the significances in the electron-channel are not very promising for the cut-based cumulative selections. To find better significances, we exploited some kind of optimization technique. First of all, we select the following kinematical variable: $\eta_{l}$ (rapidity of the forward lepton); $\Delta \eta_{j l}=\eta_{j_{f}}-\eta_{l_{f}}$ (the rapidity differences between the forward jet and forward lepton) $; m_{\phi j}$ (three jet invariant masses, i.e., two Higgs-boson candidate jets and one forward jet); $H_{T}$ (the scalar sum of the transverse momentum of of jets); $\left|\vec{H}_{T}\right|$ (the vector sum of the transverse momentum of of jets). The optimization starts with the events which passed the forward jet criterion (after selection criterion e). The numerical values of all these kinematical variables are varied within a maximum and minimum ranges (by seeing the corresponding distributions). In particular, we varied the following kinematical ranges: upper values of $\eta_{l}$ in the range $(1.0,2.5)$ with step-size 0.1 and the lower values in $(-2.5,-1.0)$ with 

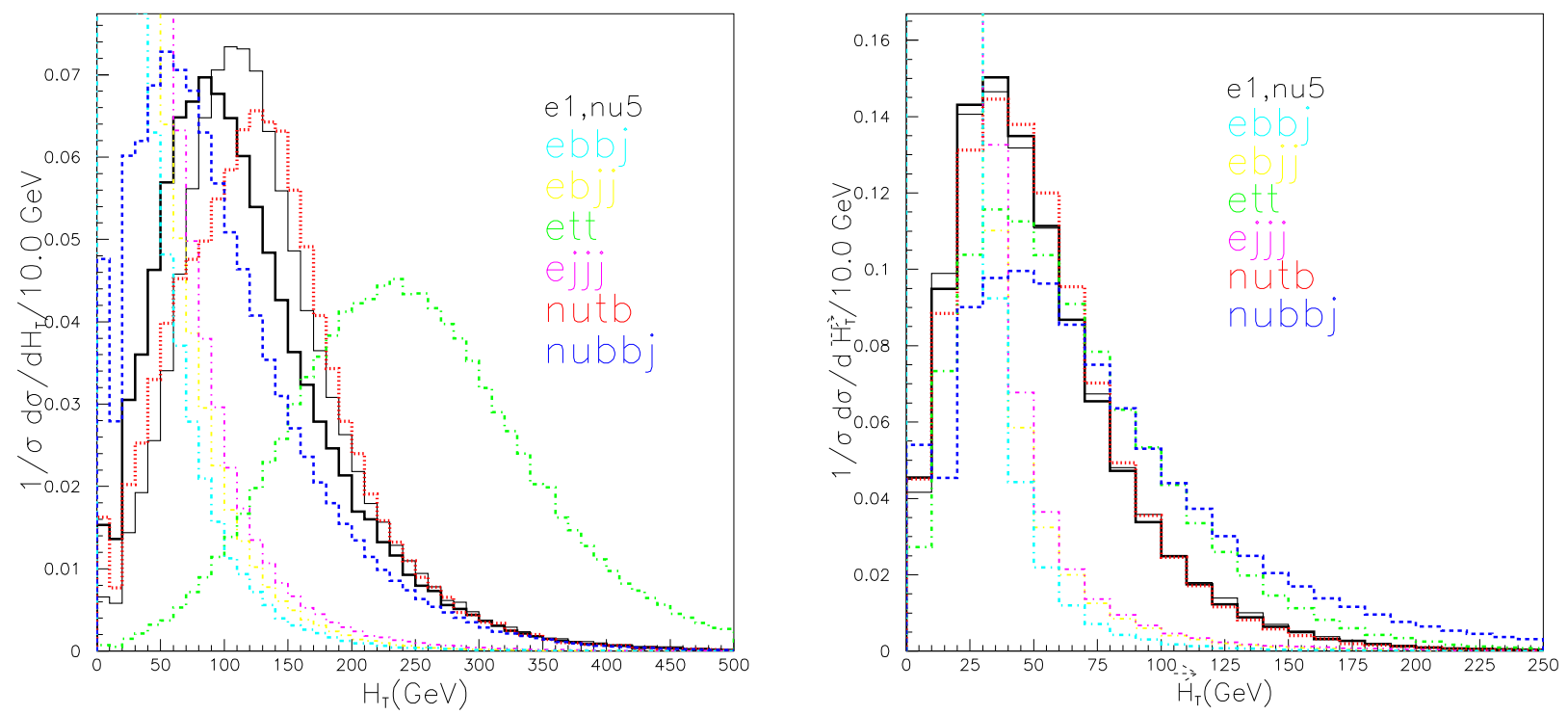

Figure 14: Left-panel: $H_{T}=\sum\left|P t_{j}\right|$ distribution for $e 1$ benchmark and $\nu 5$ benchmark. The distributions for other signal benchmarks in both the channels are somewhat identical. Rightpanel: $\vec{H}_{T}=\left|\sum \vec{P} t_{j}\right|$ defined (in the figure the magnitude of the vector is naturally implied) in kinematical selection (i), for the leptonic and missing energy channels (the presence of lepton or $\mathbb{F}_{T}$ do not matter directly for the $\vec{H}_{T}$ distribution, however the number of jets does.) for $e 1$ and $\nu 5$ benchmark points. The distributions for other benchmarks in both the signal channels are somewhat similar.

step-size 0.1 ; the upper values of $\Delta \eta_{j l}$ in $(0.0,1.5)$ with step-size 0.1 , while the lower values in $(-6.0,-3.0)$ with step-size $0.1 ; m_{\phi j}$ in $(80.0,180.0)$ with step-size $10.0 ; H_{T}$ in $(70.0,140.0)$ with step-size 10.0 ; and $\left|\vec{H}_{T}\right|$ in $(30.0,60.0)$ with step-size 10.0. We have checked approximately 44695552 numbers of combinations and estimated the numbers of signal events and the total SM backgrounds and finally the significances.

The kinematical selection configurations and their numerical values for which the significances (in the fifth and ninth column) for integrated luminosity of $100 \mathrm{fb}^{-1}$ are maximum is shown in Table 5. It turns out that for Higgs boson masses up to $82.0 \mathrm{GeV}$, the significances reach at approximately $2.1 \sigma$. In the right panel, we demanded number of signal events at least 10 for the low luminosity option (for Higgs masses greater than $80.0 \mathrm{GeV}$, we found approximately 5 signal events) and for that the number of backgrounds is also large. It is clear that for the Higgs boson masses of approximately $65.0 \mathrm{GeV}$, the significances could be $0.60(2.0) \sigma$ for $100(1000) \mathrm{fb}^{-1}$.

As the above neutral current signal, discussed above, does not have large significances in simple cut-based selection, we turn our attention to the charged current mode. It is clear from the right panel of Fig 5 that the events rates are high enough to consider this case. 


\subsection{2 $\mathbb{E}_{T}+3$-jet: missing-energy channel}

We are now looking for the feasibility of finding the Higgs boson in $\mathbb{E}_{T}+3$-jet. Like in the $e+3$-jet discussed in the previous sections, we apply kinematical cuts to isolate the beyond SM-type Higgs boson signal from the backgrounds. These cuts are, however, slightly different. The number of signal and SM background events are tabulated in Table 6 .

- A: We first selected events containing at least three jets, i.e., $N_{\text {jet }} \gtrsim 3$. The distribution of the number of jets $\left(N_{\text {jet }}\right)$ is shown (only for the $\nu 5$ benchmark) in the left panel of Fig.8. The jet reconstruction criterion are same as of the $e+3$-jet channel - thus the jet efficiencies of the SM backgrounds are exactly same. The more massive the Higgs, the larger are the jet efficiencies as it is evident from the Table 6.

- B: We required no presence of an electron in our event. If we find an electron with $p_{T}>$ 15.0 GeV and $\eta<3.0$, we reject such events, i.e., we are vetoing. This selection largely reduced the SM backgrounds as compared to the case with an explicit electron. For example, ebbj, ebjj and ejjj are reduced by approximately $77 \%, 75 \%$ and $80 \%$, while for ett the reduction is close to $86.7 \%$.

The $\nu j j j, \nu b j j$ and $\nu b b j$ are reduced by approximately $0.2 \%, 1.0 \%$ and $3.0 \%$, respectively. For the $\nu b b j$ case having an secondary electron from $B$-meson semileptonic decay is more probable than in $\nu b j j$ and much more than in $\nu j j j$. Such an expectation is, indeed, confirmed. The efficiencies of this selections for all the five signal benchmarks are nearly identical (approximately 95.5\%) as this is not related directly with the Higgs boson masses.

- C: Like in the $e+3$-jet channel in the previous section, here also we demanded at least two $b$-tagged jets with the inclusion of a proper mis-tagging. The distributions of the number of $b$-tagged jets $\left(N_{b-t a g}\right)$ are shown in the right panel of Fig. 8. The efficiencies for the missing-energy signals and SM-backgrounds are similar to the $e+3$-jet channel - as the main difference between these two channels is the presence of an electron and missing energy.

- D: After identifying the $b$-tagged jet with proper mis-tagging, like in the leptonic channel, we follow exactly similar procedure to reconstruct the non-SM Higgs boson $\left(m_{h_{1}}\right)$ masses. The reconstructed di-bjet invariant mass, $m_{b b}$ tends to peak in the lower values than the benchmark values. Due to this the mass window selection reduces the number of events more for higher Higgs boson mass. As it is clear from Table 6 , for the $\nu 1-\nu 5$ benchmarks cases the efficiencies are approximately $56.1 \%, 49.5 \%, 38.0 \%, 35.1 \%$ and $26.6 \%$. They are decreasing (as mentioned earlier) with the increase of the Higgs boson masses in the respective benchmark points. The $\nu b b j$ background survived by approximately, $28.4 \%$, $14.7 \%, 9.9 \%, 9.0 \%$ and $6.1 \%$ for the $\nu 1-\nu 5$ benchmark respectively - follow similar pattern like the signal benchmarks. We also find the same patterns for ebbj background $-26.2 \%, 25.4 \%, 19.2 \%, 18.3 \%$ and $15.2 \%$ (as the mass window selection depends upon the masses of the Higgs boson in the respective benchmarks). In addition, the ebbj background has a Z-exchange diagram, i.e., $e Z j$ with $Z \rightarrow b \bar{b}$. For the benchmark points where the Higgs boson is close to the Z-boson mass, i.e., for $\nu 4$ and $\nu 5$, the reduction is 
maximal. For $e b j j$ the events survived are approximately, 27.4\%, 27.7\%, 18.1\%, 19.3\% and $12.0 \%$ a for $\nu 1-\nu 5$ benchmarks respectively, somewhat similar to ebbj. For ebjj two more issues are important to mention, the $e b Z$ with $Z \rightarrow j \bar{j}$ and mis-tagging of jets. For $\nu t b$, the event survived are approximately, $19.0 \%$ for all the benchmark points as many effects play together, e.g., top-quark decay, mistagging, differences between Higgs boson and W-boson masses in those benchmark points.

- E: Like in the electron channel selection (e), here we also demanded the presence of one forward jet. All the signal benchmarks survived by approximately 99\%. This criterion has a mild overall impact - it rejects the irreducible backgrounds $\nu b b j(\nu b j j)$ by approximately $34.5 \%, 21.0 \%, 17.4 \%, 16.7 \%$ and $21.7 \%(22.9 \%, 33.0 \%, 33.4 \%, 33.4 \%$ and $29.0 \%)$ for the $\nu 1-\nu 5$ benchmarks respectively. The background $\nu j j j$ is reduced by approximately from $16.0 \%-25.0 \%$ from $\nu 1$ to $\nu 5$ benchmark points.

- F: The signal contains now a neutrino and this explicitly leads to missing energy (other than the jet-energy smearing and mis-measurements). The $\mathbb{F}_{T}$ is shown in the right panel of Fig.9. We demanded that $\mathbb{F}_{T}$ should be larger than $15 \mathrm{GeV}$. All the signal benchmark survived by $91 \%$ or more. This criterion suppressed all the background except for ett. Most importantly, at this stage ejjj turns out to be zero. The ebjj is reduced by $65 \%$ $35 \%$, while ebbj is reduced by $65 \%-55 \%$ for all the signal benchmarks. The relatively larger reduction in $e b b j$ is again due to the semi-leptonic decays of the two bottom quarks. The backgrounds $\nu b b j$ survived by $99 \%$ for $\nu 1$ benchmarks and for all other benchmarks it survived by more than $96 \%$ while $\nu b j j(\nu j j j)$ is survived by approximately $96 \%(99 \%)$.

- G: With the identification of forward jet we reconstructed the three jet invariant mass of the $m_{b b j_{f}}$. The distribution is shown in Fig 13 for the $\nu 5$ benchmark. All other benchmarks have similar distributions with the peaks nearly at the same values around $120 \mathrm{GeV}$. Furthermore, we demanded that $m_{b b j_{f}}>210 \mathrm{GeV}$. The signal reduces by approximately $50 \%$ to $45 \%$ (from $\nu 1$ to $\nu 5$ benchmark points) while the background reduces severely, e.g., $\nu b b j$ reduces by approximately, $80 \%$. The other irreducible backgrounds, $\nu t b, e b b j$ and ett reduce by approximately 95\%, 70\%-80\% and 96\%-93\%, respectively for all the signal benchmarks.

- H: Like in the $e+3$-jet channel, we demanded $H_{T}>100 \mathrm{GeV}$. The event efficiencies for the signal and all backgrounds follow similar pattern for the lepton signal channel. This is due to the fact that this variable dependents on the number of jets and their transverse momentum and does not have an explicit dependence on neither electron nor neutrino. Except ebbj (reduced by approximately 30\%) this selection hardly affects any other backgrounds.

At this stage, for all the signal benchmarks, the backgrounds of $\nu t b$ and $\nu j j j$ are of similar size to the signal events while ebbj is slightly more than factor of two. The signals events are quite large and the SM-backgrounds are manageable. This leads to quite high significances to observe the Higgs boson signal in the missing energy channel.

- I: However, to reduce the ebbj further we invoked the magnitude of $\left|\vec{H}_{T}\right|>50 \mathrm{GeV}$. The signals and $\nu t b$ are reduced approximately 50\% while ebbj (except the $\nu 1$ benchmark, where it reduced by $85 \%$ ) goes to zero. 
After applying the cumulative selection from (A)-(I), we estimated the total SM background events to be found in the final column of Table 6. The significance for $100 \mathrm{fb}^{-1}$ integrated luminosity for $\nu 1, \nu 2, \nu 3, \nu 4$ and $\nu 5$ benchmark points are is $3.3 \sigma, 1.8 \sigma, 1.5 \sigma, 1.8 \sigma$ and $1.4 \sigma$, respectively. Thus except the first benchmark, with Higgs boson mass around $66.0 \mathrm{GeV}$, all other benchmarks are not good enough to be observed with a high confidence level with $100 \mathrm{fb}^{-1}$. We find that with $1000 \mathrm{fb}^{-1}$, the significances of those benchmarks can be found with $10.6 \sigma$, $5.8 \sigma, 4.9 \sigma, 5.5 \sigma$ and $4.5 \sigma$. With our choices of simple cut-based selections and at the end of the LHeC, a $5.0 \sigma$ discovery would be possible for Higgs boson masses up to $90 \mathrm{GeV}$.

Compared to the electron channel, the overall significances in the missing energy channel are higher for relatively large Higgs masses. However, likewise in the electron channel, here we have exploited the optimization by varying four different kinematical parameters, $\mathbb{F}_{T}, m_{\phi j}, H_{T}$ and the magnitude of $\vec{H}_{T}$. The optimization starts after the events have passed the forward jet criterion (after selection criterion $\mathrm{E}$ ). The numerical values of all these kinematical variables are varied within a maximum and minimum range (by seeing the corresponding distributions). We varied the $\mathbb{H}_{T}$ in the ranges $(10.0,40.0)$ with step-size 5.0 , together with $m_{\phi j}$, $H_{T}$ and the magnitude of $\vec{H}_{T}$ used in the electron channel with the same ranges. We have checked approximately 2464 number of combinations and estimated the number of signal events and the total SM backgrounds and finally the significances. The kinematical configurations and their numerical values for which the significances (in the fifth and ninth column) for $100 \mathrm{fb}$ ${ }^{-1}$ are maximum is shown in Table [7. It turns out that one can have $2.4 \sigma$ up to Higgs boson masses of $88 \mathrm{GeV}$. The significances in the parenthesis are for $1000 \mathrm{fb}^{-1}$. It turns out that the Higgs boson with masses more than 90 can have significances approximately $5 \sigma$.

\section{Conclusions}

The discovery of the SM-like Higgs boson at LHC establishes the correct pattern of the electroweak symmetry breaking with a doublet. However, to overcome theoretical shortcomings, multiple doublets are naturally introduced in models beyond the SM - both without and with supersymmetry. All such multiple doublet models predict the presence of more Higgses with different mass ranges. Hence looking for any kind of such Higgses in the present and upcoming collision experiments is a good opportunity to probe particle physics beyond the SM.

Among the experimental facilities which will soon become operational and potentially competitive to look for the intermediate mass Higgs bosons, is the LHeC facility (with ep collisions) located at CERN expected to be operational in 2020.

In this work, we have considered the NMSSM model where the non-SM type Higgs boson with masses less than the SM-like Higgs boson is naturally possible with all the theoretical and most up-to-date experimental constraints from the low energy experiments as well as the supersymmetry and Higgs searches results from the LHC. The model has naturally a low mass lightest neutralino $\left(\tilde{\chi}_{1}^{0}\right)$ which serves as the possible candidate of the cold-dark matter. Apart from the particle physics constraints mentioned above, our parameter space respects all the dark matter constraints, including WMAP and dark matter searches.

We have considered two different production mechanisms of this intermediate Higgs boson, namely, $e h_{1} q_{f}$ and $\nu h_{1} q_{f}$, and used the $h_{1} \rightarrow b \bar{b}$ decay mode to find the Higgs boson signal. In our analysis, we performed a detail parameter space scanning using NMSSMTools v.5.0.1 
assuming that the second intermediate mass Higgs boson is of the SM-type. For the allowed parameters we estimated the production cross-section and the branching ratio to find the event rate at the $\mathrm{LHeC}$ facility.

The production processes under consideration are $e+3$-jets and $\mathbb{E}_{T}+3$-jets. Two jets can originate from the Higgs boson decay, $h_{1} \rightarrow b \bar{b}$ and we demanded both of them to be $b$-tagged (with proper mis-tagging from light-flavor and gluon jets) in the central rapidity region. The remaining jet originates from the remnant of the proton fluxes which is likely to be with large rapidity (in the forward or backward region). We considered the reducible and irreducible SM backgrounds (with charge-conjugation wherever appropriate) for the charge-current processes: $\nu t b, \nu b b j, \nu b j j$ and $\nu j j j$ and neutral current processes: ebbj, ebjj, ett and ejjj.

We performed a full hadron-level Monte Carlo simulation using MadGraph/MadEvent followed by PYTHIA as the parton shower/hadronization event generator and its PYCELL toy calorimeter in accordance with the LHeC detector parameters. We carefully implemented $b$-tagging, including mis-tagging of $c$-jets, light-flavor and gluon jets.

In both of the Higgs signal under consideration, we first applied the basic event characteristics, like number of jets, number of lepton, missing energy profile, number of $b$-tagged jets. The kinematics for both the signals are very interesting due to the fact that the Higgs boson is produced in the central rapidity region such that the two $b$-tagged jets are also central. We reconstructed the invariant mass of these two (or more) $b$-tagged jets and identified the best combinations where the reconstructed Higgs boson mass is close to the benchmark values and thereafter selected events only with slightly asymmetric choices with $m_{h_{1}}-15<m_{b b}<m_{h_{1}}+5$ $\mathrm{GeV}$. This selection reduces the SM backgrounds to a large extent and the invariant mass ensures the finding of the Higgs boson.

As a next step of our selection, by seeing the rapidity profiles, we identified the most energetic light-flavor forward jet $\left(j_{f}\right)$ and demanded that the rapidity product of the forward lepton, i.e., electron $\left(j_{e}\right)$ and forward jet should be negative, i.e., they must lie in the opposite hemisphere. Furthermore, we demanded that the rapidity gap between this forward jet and forward electron should satisfy $-5.5<\Delta \eta_{j-e f}<0.5$. After this selection we calculated the three-jet invariant masses, $m_{b b j_{f}}$ which essentially gives the overall energy scale of the hard scattering. We demanded that this should be larger than $190 \mathrm{GeV}$ and this selection helps to suppress backgrounds coming from ebbj, ejjj, $\nu t b$ and ett.

After that, for the $e+3 j$ signal channel we applied some $H_{T}$ selection to suppress the SM backgrounds further. This is not enough though to ensure a relatively high confidence level. Hence finally we exploited the $\vec{H}_{T}$ which leads to better significances, but not at the discovery level though, for the intermediate non-SM type Higgs boson.

In the $e+3 j$ channel, which is naturally event rate limited, we found that to isolate the non-SM like Higgs signals, we can attain at most $0.4 \sigma$ with $100 \mathrm{fb}^{-1}$ for the $e 1$ benchmark. With ten times more luminosity, for the same benchmark with $m_{h}=63.59 \mathrm{GeV}$, we would have the significance approximately $1.3 \sigma$.

In the $\mathbb{F}_{T}+3 j$ channel with large event rate we can probe a much higher Higgs mass. We find that with $100 \mathrm{fb}^{-1}$ for the $\nu 1$ benchmark the significance would be approximately $3.3 \sigma$. For the $\nu 4$ benchmark (same as $e 5$ benchmark) we can have the significance approximately $1.8 \sigma$. With $1000 \mathrm{fb}^{-1}$ for $\nu 4$ benchmark, with Higgs masses $88.1 \mathrm{GeV}$, we would have $5.5 \sigma$. And for $\nu 5$ benchmark, with Higgs masses of $100.5 \mathrm{GeV}$ we found the significances of $4.5 \sigma$. Using a mere interpolation we would expect that one can have $5.0 \sigma$ significance up to the Higgs 
masses of around $90 \mathrm{GeV}$.

It should be noted that we have adopted a simple cut-based selection. If one would instead invoke more complex discriminators and/or use multi-variate analysis, we expect that the significance would be larger even with low luminosity option.

We have introduced a simple cut-based optimization method to enhance the Higgs boson mass reach in both the channels under consideration. In the electron channel, by varying the most important kinematical variables, $\eta_{l}, \Delta \eta_{j l}, m_{\phi j}, H_{T}$ and the magnitude of $\vec{H}_{T}$ with the maximum and minimum ranges (by inspecting the respective distributions) we optimized the significances. We found that for 100 (1000) $\mathrm{fb}^{-1}$ integrated luminosity, we can have the significances of $2.1 \sigma(6.6 \sigma)$ for the $e 4$ benchmark point with the Higgs boson masses of 82.2 $\mathrm{GeV}$.

In the missing energy channel, by varying $\mathbb{F}_{T}, m_{\phi j}, H_{T}$ and magnitude of $\vec{H}_{T}$, for $100 f b^{-1}$ integrated luminosity, we can have the significances of $2.4 \sigma(1.2 \sigma)$ for the $\nu 4(\nu 5)$ benchmark point with Higgs boson masses of $88.1(100.5) \mathrm{GeV}$. With high luminosity option one would expect the $5 \sigma$ discovery at-least up to Higgs boson masses of $95 \mathrm{GeV}$.

To conclude, after the first few years of the LHeC run, and using more complex discriminators and using multi-variate analyses, we expect that in the $e+3 j$ channel, non-SM type Higgs boson would be probed up to $85-90 \mathrm{GeV}$. In the $\mathbb{F}_{T}+3 j$ one can extend the reach at-least up to $95 \mathrm{GeV}$.

\section{Acknowledgments}

We are very much grateful for discussions with C. Hugonie and U. Ellwanger on NMSSMTools v5.0.1 and O. Mattelaer on MadGraph/MadEvent v2.4.3. We are acknowledging the High Performance Computing (HPC) facility at Uniandes and J. P. Mallarino for many useful suggestion regarding HPC facility.

\section{References}

[1] G. Aad et al. [ATLAS Collaboration], Phys. Lett. B 716 (2012) 1.

[2] S. Chatrchyan et al. [CMS Collaboration], Phys. Lett. B 716 (2012) 30.

[3] J. D. Wells, arXiv:0909.4541 [hep-ph] ; I. P. Ivanov, arXiv:1702.03776 [hep-ph].

[4] C. Grojean, CERN-PH-TH-2006-172.

[5] P. S. Bhupal Dev, C. H. Lee and R. N. Mohapatra, J. Phys. Conf. Ser. 631, no. 1, 012007 (2015) doi:10.1088/1742-6596/631/1/012007 arXiv:1503.04970 [hep-ph]].

[6] G. Seidl, hep-ph/0409162 ; C. Csaki, hep-ph/0412339.

[7] G. C. Branco, P. M. Ferreira, L. Lavoura, M. N. Rebelo, M. Sher and J. P. Silva, Phys. Rept. 516, 1 (2012) arXiv:1106.0034 [hep-ph]].

[8] J. F. Gunion and H. E. Haber, Phys. Rev. D 67, 075019 (2003) hep-ph/0207010. 
[9] A. Cordero-Cid, J. Hernandez-Sanchez, C. G. Honorato, S. Moretti, M. A. Perez and A. Rosado, JHEP 1407, 057 (2014) arXiv:1312.5614 [hep-ph]]; O. Felix-Beltran, F. Gonzalez-Canales, J. Hernandez-Sanchez, S. Moretti, R. Noriega-Papaqui and A. Rosado, Phys. Lett. B 742, 347 (2015) arXiv:1311.5210 [hep-ph]]; J. HernandezSanchez, S. Moretti, R. Noriega-Papaqui and A. Rosado, JHEP 1307, 044 (2013) arXiv:1212.6818; J. Hernandez-Sanchez, S. Moretti, R. Noriega-Papaqui and A. Rosado, PoS CHARGED 2012, 029 (2012) arXiv:1302.0083]; J. Hernandez-Sanchez, C. G. Honorato, M. A. Perez and J. J. Toscano, Phys. Rev. D 85, 015020 (2012) arXiv:1108.4074 [hep-ph]]; J. L. Diaz-Cruz and J. J. Toscano, Phys. Rev. D 62, 116005 (2000) hep-ph/9910233]; A. G. Akeroyd, S. Moretti and J. Hernandez-Sanchez, arXiv:1409.7596 [hep-ph];

[10] G. L. Kane, C. F. Kolda, L. Roszkowski and J. D. Wells, Phys. Rev. D 49, 6173 (1994) doi:10.1103/PhysRevD.49.6173 hep-ph/9312272.

[11] H.-P. Nilles, M. Srednicki and D. Wyler, Phys. Lett. B 120 (1983) 346; J.P. Derendinger and C.A. Savoy, Nucl. Phys. B 237 (1984) 307; J.R. Ellis, J.F. Gunion, H.E. Haber, L. Roszkowski, and F. Zwirner, Phys. Rev. D 39 (1989) 844; U. Ellwanger, Phys. Lett. B 303 (1993) 271.

[12] M. Drees, Int. J. Mod. Phys. A 4, 3635 (1989). doi:10.1142/S0217751X89001448

[13] F. Franke and H. Fraas, Int. J. Mod. Phys. A 12, 479 (1997) doi:10.1142/S0217751X97000529 hep-ph/9512366.

[14] M. Maniatis, Int. J. Mod. Phys. A 25, 3505 (2010) doi:10.1142/S0217751X10049827 arXiv:0906.0777 [hep-ph]].

[15] U. Ellwanger, C. Hugonie and A. M. Teixeira, Phys. Rept. 496, 1 (2010) doi:10.1016/j.physrep.2010.07.001 arXiv:0910.1785 [hep-ph]].

[16] D. J. Miller, S. Moretti and R. Nevzorov, hep-ph/0501139.

[17] R. Barbieri, D. Buttazzo, K. Kannike, F. Sala and A. Tesi, Phys. Rev. D 87, no. 11, 115018 (2013) doi:10.1103/PhysRevD.87.115018 [arXiv:1304.3670 [hep-ph]]; M. Farina, M. Perelstein and B. Shakya, JHEP 1404, 108 (2014) doi:10.1007/JHEP04(2014)108 arXiv:1310.0459 [hep-ph]].

[18] J. E. Kim and H. P. Nilles, Phys. Lett. B 138 (1984) 150.

[19] J. R. Ellis, J. F. Gunion, H. E. Haber, L. Roszkowski and F. Zwirner, Phys. Rev. D 39 (1989) 844.

[20] For e.g., P. Bechtle, H. E. Haber, S. Heinemeyer, O. Stl, T. Stefaniak, G. Weiglein and L. Zeune, arXiv:1608.00638 [hep-ph]; J. Quevillon, arXiv:1405.2241 [hep-ph]; B. Dumont, J. F. Gunion and S. Kraml, Phys. Rev. D 89 (2014) 055018. 
[21] P. Drechsel, L. Galeta, S. Heinemeyer and G. Weiglein, arXiv:1601.08100 [hep-ph]; P. Drechsel, DESY-THESIS-2016-019; F. Staub, P. Athron, U. Ellwanger, R. Grober, M. Muhlleitner, P. Slavich and A. Voigt, Comput. Phys. Commun. 202, 113 (2016) doi:10.1016/j.cpc.2016.01.005 [arXiv:1507.05093 [hep-ph]].

[22] M. D. Goodsell and F. Staub, arXiv:1604.05335 [hep-ph].

[23] U. Ellwanger, J. F. Gunion, C. Hugonie and S. Moretti, hep-ph/0401228; V. Barger, P. Langacker, H. S. Lee and G. Shaughnessy, Phys. Rev. D 73, 115010 (2006) doi:10.1103/PhysRevD.73.115010 hep-ph/0603247]; D. J. Miller, R. Nevzorov and P. M. Zerwas, Nucl. Phys. B 681, 3 (2004) doi:10.1016/j.nuclphysb.2003.12.021 hep-ph/0304049]; U. Ellwanger and M. Rodriguez-Vazquez, JHEP 1602, 096 (2016) doi:10.1007/JHEP02(2016)096 arXiv:1512.04281 [hep-ph]]; J. S. Kim, D. Schmeier and J. Tattersall, Phys. Rev. D 93, no. 5, 055018 (2016) doi:10.1103/PhysRevD.93.055018 arXiv:1510.04871 [hep-ph]]; J. Rathsman and T. Rossler, Adv. High Energy Phys. 2012, 853706 (2012) doi:10.1155/2012/853706 arXiv:1206.1470 [hep-ph]; R. Enberg, R. Pasechnik and O. Stal, Phys. Rev. D 85, 075016 (2012) doi:10.1103/PhysRevD.85.075016 arXiv:1112.4699 [hep-ph]]; J. F. Gunion, Y. Jiang and S. Kraml, Phys. Lett. B 710 (2012) 454; S. Liebler, H. Mantler and M. Wiesemann, arXiv:1608.02949 [hep-ph]; M. Carena, H. E. Haber, I. Low, N. R. Shah and C. E. M. Wagner, Phys. Rev. D 93, no. 3, 035013 (2016) doi:10.1103/PhysRevD.93.035013 arXiv:1510.09137 [hep-ph]]; E. Conte, B. Fuks, J. Guo, J. Li and A. G. Williams, JHEP 1605, 100 (2016) doi:10.1007/JHEP05(2016)100 [arXiv:1604.05394 [hep-ph]]; S. F. King, M. Muhlleitner, R. Nevzorov and K. Walz, Nucl. Phys. B 870 (2013) 323 ; S. F. King, M. Muhlleitner and R. Nevzorov, Nucl. Phys. B 860 (2012) 207 ;N. D. Christensen, T. Han, Z. Liu and S. Su, JHEP 1308 (2013) 019 ; J. Cao, F. Ding, C. Han, J. M. Yang and J. Zhu, JHEP 1311 (2013) 018; G. Belanger, U. Ellwanger, J. F. Gunion, Y. Jiang, S. Kraml and J. H. Schwarz, JHEP 1301 (2013) 069; U. Ellwanger, J. F. Gunion, C. Hugonie and S. Moretti, hep-ph/0305109; D. Das, U. Ellwanger and A. M. Teixeira, JHEP 1204, 067 (2012) doi:10.1007/JHEP04(2012)067 arXiv:1202.5244 [hep-ph]]; U. Ellwanger and C. Hugonie, arXiv:1203.5048 [hep-ph]; K. S. Jeong, Y. Shoji and M. Yamaguchi, JHEP 1411, 148 (2014) doi:10.1007/JHEP11(2014)148 arXiv:1407.0955 [hep-ph]]; B. Dutta, Y. Gao and B. Shakya, Phys. Rev. D 91, no. 3, 035016 (2015) doi:10.1103/PhysRevD.91.035016 [arXiv:1412.2774 [hep-ph]]; S. Baum, K. Freese, N. R. Shah and B. Shakya, arXiv:1703.07800 [hep-ph].

[24] H. K. Dreiner, F. Staub and A. Vicente, Phys. Rev. D 87, no. 3, 035009 (2013) doi:10.1103/PhysRevD.87.035009 [arXiv:1211.6987 [hep-ph]].

[25] U. Ellwanger, JHEP 1311, 108 (2013) doi:10.1007/JHEP11(2013)108 arXiv:1309.1665 [hep-ph]].

[26] F. Domingo and G. Weiglein, JHEP 1604, 095 (2016) doi:10.1007/JHEP04(2016)095 arXiv:1509.07283 [hep-ph]].

[27] D. Barducci, G. Belanger, C. Hugonie and A. Pukhov, JHEP 1601, 050 (2016) doi:10.1007/JHEP01(2016)050 [arXiv:1510.00246 [hep-ph]]. 
[28] N. E. Bomark, S. Moretti, S. Munir and L. Roszkowski, PoS EPS -HEP2015, 162 (2015) arXiv:1510.02661[hep-ph]];N. E. Bomark, S. Moretti, S. Munir and L. Roszkowski, JHEP 1502, 044 (2015) doi:10.1007/JHEP02(2015)044 arXiv:1409.8393 [hep-ph]]; S. Moretti and S. Munir, Adv. High Energy Phys. 2015, 509847 (2015) doi:10.1155/2015/509847 arXiv:1505.00545 [hep-ph]]; M. M. Almarashi and S. Moretti, Phys. Rev. D 85, 017701 (2012) doi:10.1103/PhysRevD.85.017701 arXiv:1109.1735 [hep-ph]]; M. Almarashi and S. Moretti, Phys. Rev. D 84, 015014 (2011) doi:10.1103/PhysRevD.84.015014 arXiv:1105.4191 [hep-ph]]; N. E. Bomark, S. Moretti, S. Munir and L. Roszkowski, PoS Charged 2014, 029 (2015) arXiv:1412.5815 [hep-ph]]; N. E. Bomark, S. Moretti and L. Roszkowski, J. Phys. G 43, no. 10, 105003 (2016) doi:10.1088/0954-3899/43/10/105003 arXiv:1503.04228 [hep-ph]]; M. M. Almarashi and S. Moretti, Phys. Rev. D 83, 035023 (2011) doi:10.1103/PhysRevD.83.035023 [arXiv:1101.1137 [hep-ph]].

[29] C. T. Potter, Eur. Phys. J. C 76, no. 1, 44 (2016) doi:10.1140/epjc/s10052-015-3867-x arXiv:1505.05554 [hep-ph]].

[30] M. Guchait and J. Kumar, Int. J. Mod. Phys. A 31, no. 12, 1650069 (2016) doi:10.1142/S0217751X1650069X [arXiv:1509.02452 [hep-ph]]; M. Guchait and J. Kumar, arXiv:1608.05693 [hep-ph].

[31] V. Khachatryan et al. [CMS Collaboration], JHEP 1601, 079 (2016) doi:10.1007/JHEP01(2016)079 [arXiv:1510.06534 [hep-ex]].

[32] https://lhec.web.cern.ch

[33] See for e.g., M.Klein, R.Yoshida: Collider Physics at HERA Prog.Part.Nucl.Phys. 61(2008)343-393.

[34] T. Han and B. Mellado, Phys. Rev. D 82, 016009 (2010) arXiv:0909.2460 [hep-ph]].

[35] I. A. Sarmiento-Alvarado, A. O. Bouzas and F. Larios, arXiv:1412.6679 [hep-ph] ; A. O. Bouzas and F. Larios, J. Phys. Conf. Ser. 651, no. 1, 012004 (2015). doi:10.1088/1742-6596/651/1/012004

[36] S. P. Das, J. Hernandez-Sanchez, S. Moretti, A. Rosado and R. Xoxocotzi, Phys. Rev. D 94, no. 5, 055003 (2016) doi:10.1103/PhysRevD.94.055003 [arXiv:1503.01464 [hep-ph]].

[37] M. Drees and G. Gerbier, arXiv:1204.2373 [hep-ph].

[38] J. C. Sanabria, Rev. Acad. Colomb. Cienc. 38, 34 (2014).

[39] Planck Collaboration, P. Ade et al., Planck 2015 results. XIII. Cosmological parameters, http://arxiv.org/abs/1502.01589 arXiv:1502.01589.

[40] G. Belanger, F. Boudjema, C. Hugonie, A. Pukhov and A. Semenov, JCAP 0509, 001 (2005) doi:10.1088/1475-7516/2005/09/001 hep-ph/0505142; J. Cao, Y. He, L. Shang, W. Su, P. Wu and Y. Zhang, JHEP 1610, 136 (2016) doi:10.1007/JHEP10(2016)136 arXiv:1609.00204 [hep-ph]]; J. Cao, Y. He, L. Shang, W. Su and Y. Zhang, JHEP 1608, 
037 (2016) doi:10.1007/JHEP08(2016)037 arXiv:1606.04416 [hep-ph]]; D. Das and U. Ellwanger, JHEP 1009, 085 (2010) doi:10.1007/JHEP09(2010)085 arXiv:1007.1151 [hepph]]; Q. F. Xiang, X. J. Bi, P. F. Yin and Z. H. Yu, arXiv:1606.02149 [hep-ph]; J. Kozaczuk and S. Profumo, Phys. Rev. D 89, no. 9, 095012 (2014) doi:10.1103/PhysRevD.89.095012 arXiv:1308.5705 [hep-ph]].

[41] M. Badziak, M. Olechowski and P. Szczerbiak, JHEP 1603, 179 (2016) doi:10.1007/JHEP03(2016)179 [arXiv:1512.02472 [hep-ph]].

[42] S. Horiuchi, O. Macias, D. Restrepo, A. Rivera, O. Zapata and H. Silverwood, JCAP 1603, no. 03, 048 (2016) doi:10.1088/1475-7516/2016/03/048 arXiv:1602.04788 [hep$\mathrm{ph}]$.

[43] J. D. Ruiz-Alvarez, C. A. de S.Pires, F. S. Queiroz, D. Restrepo and P. S. Rodrigues da Silva, Phys. Rev. D 86, 075011 (2012) doi:10.1103/PhysRevD.86.075011 arXiv:1206.5779 [hep-ph]].

[44] S. P. Das, M. Guchait and D. P. Roy, Phys. Rev. D 90, no. 5, 055011 (2014) doi:10.1103/PhysRevD.90.055011 [arXiv:1406.6925 [hep-ph]].

[45] A. Florez, L. Bravo, A. Gurrola, C. Avila, M. Segura, P. Sheldon and W. Johns, Phys. Rev. D 94, no. 7, 073007 (2016) doi:10.1103/PhysRevD.94.073007 [arXiv:1606.08878 [hep-ph]].

[46] P. Diessner, J. Kalinowski, W. Kotlarski and D. Stoeckinger, JHEP 1603, 007 (2016) doi:10.1007/JHEP03(2016)007 arXiv:1511.09334 [hep-ph]].

[47] K. Hagimoto, T. Kobayashi, H. Makino, K. i. Okumura and T. Shimomura, JHEP 1602, 089 (2016) doi:10.1007/JHEP02(2016)089 [arXiv:1509.05327 [hep-ph]].

[48] C.-C. Jean-Louis and G. Moreau, J. Phys. G 37, 105015 (2010) doi:10.1088/0954-3899/37

[49] U. Ellwanger, J. F. Gunion and C. Hugonie, JHEP 0502 (2005) 066.

[50] D. Barducci, G. Belanger, J. Bernon, F. Boudjema, J. DaSilva, S. Kraml, U. Laa, A. Pukhov, arXiv:1606.03834 [hep-ph]

[51] G. Belanger, F. Boudjema, A. Pukhov and A. Semenov, Comput. Phys. Commun. 185, 960 (2014) doi:10.1016/j.cpc.2013.10.016 [arXiv:1305.0237 [hep-ph]].

[52] S. Zheng, Eur. Phys. J. C 75, no. 5, 195 (2015) doi:10.1140/epjc/s10052-015-3416-7 arXiv:1405.6907 [hep-ph]].

[53] J. C. Sanabria [ATLAS and CMS Collaborations], Nucl. Part. Phys. Proc. 267-269, 25 (2015). doi:10.1016/j.nuclphysbps.2015.10.078

[54] G. Aad et al. [ATLAS and CMS Collaborations], JHEP 1608, 045 (2016) doi:10.1007/JHEP08(2016)045 arXiv:1606.02266 [hep-ex]].

[55] V. Khachatryan et al. [CMS Collaboration], JHEP 1702, 135 (2017) doi:10.1007/JHEP02(2017)135 [arXiv:1610.09218 [hep-ex]]. 
[56] G. Aad et al. [ATLAS Collaboration], JHEP 1511, $206 \quad$ (2015) doi:10.1007/JHEP11(2015)206 arXiv:1509.00672 [hep-ex]].

[57] A. Butter, T. Plehn, M. Rauch, D. Zerwas, S. Henrot-Versill and R. Lafaye, Phys. Rev. D 93, 015011 (2016) doi:10.1103/PhysRevD.93.015011 [arXiv:1507.02288 [hep-ph]].

[58] SLD Electroweak Group, DELPHI, ALEPH, SLD, SLD Heavy Flavour Group, OPAL, LEP Electroweak Working Group, L3 Collaboration, S. Schael et al., Precision electroweak measurements on the $Z$ resonance, Phys. Rept. 427 (2006) 257-454.

[59] Muon g-2 Collaboration, G. W. Bennett et al., Measurement of the negative muon anomalous magnetic moment to 0.7 ppm, Phys. Rev. Lett. 92 (2004) 161802.

[60] Heavy Flavor Averaging Group (HFAG) Collaboration, Y. Amhis et al., Averages of b-hadron, c-hadron, and $\tau$-lepton properties as of summer 2014, arXiv:1412.7515.

[61] BaBar Collaboration, J. P. Lees et al., Evidence of $B^{+} \rightarrow \tau^{+} \nu$ decays with hadronic B tags, Phys. Rev. D88 (2013), no. 3031102.

[62] M. Aaboud et al. [ATLAS Collaboration], Phys. Rev. D 94, no. 3, 032005 (2016) doi:10.1103/PhysRevD.94.032005 [arXiv:1604.07773 [hep-ex]].

[63] J. Alwall et al., JHEP 1407, 079 (2014) doi:10.1007/JHEP07(2014)079 arXiv:1405.0301 [hep-ph]].

[64] O. Bruening and M. Klein, Mod. Phys. Lett. A 28, no. 16, 1330011 (2013) arXiv:1305.2090 [physics.acc-ph]].

[65] J. L. Abelleira Fernandez et al. [LHeC Study Group Collaboration], "On the Relation of the LHeC and the LHC," arXiv:1211.5102 [hep-ex].

[66] J. L. Abelleira Fernandez et al. [LHeC Study Group Collaboration], "A Large Hadron Electron Collider at CERN: Report on the Physics and Design Concepts for Machine and Detector," J. Phys. G 39, 075001 (2012) arXiv:1206.2913 [physics.acc-ph]].

[67] See for e.g. R B Appleby et al 2013 J.Phys.G: Nucl.Part.Phys.40 125004.

[68] R. D. Ball et al. [NNPDF Collaboration], Nucl. Phys. B 877, 290 (2013) doi:10.1016/j.nuclphysb.2013.10.010 [arXiv:1308.0598 [hep-ph]]; R. D. Ball et al. [NNPDF Collaboration], JHEP 1504, 040 (2015) doi:10.1007/JHEP04(2015)040 arXiv:1410.8849 [hep-ph]].

[69] J. Pumplin, D. R. Stump, J. Huston, H. L. Lai, P. M. Nadolsky and W. K. Tung, JHEP 0207, 012 (2002) hep-ph/0201195.

[70] T. Sjostrand, S. Mrenna and P. Z. Skands, "PYTHIA 6.4 Physics and Manual," JHEP 0605, 026 (2006) hep-ph/0603175.

[71] R. N. Cahn and S.Dawson, Phys. Lett. B 136 (1984) 196; M. S. Chanowitz and M. K.Gaillard, Phys. Lett. B 142 (1984) 85; G. L.Kane and W. W.Repko and W. B.Rolnick, Phys. Lett. B 148 (1984) 367. 
[72] P. Agrawal, S. Bandyopadhyay and S. P. Das, arXiv:1308.6511 [hep-ph].

[73] P. Agrawal, S. Bandyopadhyay and S. P. Das, Phys. Rev. D 88, no. 9, 093008 (2013) doi:10.1103/PhysRevD.88.093008 [arXiv:1308.3043 [hep-ph]].

[74] S. P. Das and M. Drees, Phys. Rev. D 83, 035003 (2011) arXiv:1010.3701 [hep-ph]]; S. P. Das and M. Drees, J. Phys. Conf. Ser. 259, 012071 (2010) arXiv:1010.2129 [hep-ph]]; S. P. Das, A. Datta and M. Drees, AIP Conf. Proc. 1078, 223 (2009) arXiv:0809.2209 [hep-ph]]. 


\begin{tabular}{|c|c|c|c|c|c|c|c|c|c|c|c|c|}
\hline Proc, $m_{h}$ & RawEvt & $\mathrm{a}$ & $\mathrm{b}$ & $\mathrm{c}$ & d & e & $\mathrm{f}$ & $\mathrm{g}$ & $\mathrm{h}$ & $\mathrm{i}$ & $\mathrm{j}$ & $\mathcal{S}$ \\
\hline$e 1,63.59$ & 882.4 & "351.2 & "330.0 & 45.3 & 24.5 & 23.9 & 12.8 & 12.5 & 6.3 & 6.0 & 2.2 & $0.40(1.3)$ \\
\hline$e b b j$ & 2688390.0 & 176102.0 & 135388.5 & 15530.5 & 3759.5 & 3461.2 & 2655.6 & 2476.5 & 343.1 & 179.0 & 29.8 & \multirow{8}{*}{$\mathrm{B}=30.8$} \\
\hline$e b j j$ & 330834.0 & 31317.5 & 23381.6 & 575.6 & 146.4 & 110.8 & 74.4 & 73.6 & 3.3 & 2.5 & 0.8 & \\
\hline$e t \bar{t}$ & 1425.5 & 1313.8 & 1136.9 & 131.8 & 20.2 & 19.9 & 7.9 & 7.8 & 0.4 & 0.4 & 0.2 & \\
\hline$e j j j$ & 37224100.0 & 4943049.0 & 3940097.5 & 8862.8 & 1611.4 & 966.9 & 805.7 & 805.7 & 161.1 & 0.0 & 0.0 & \\
\hline$\nu b b j$ & 21385.4 & 4040.5 & 112.0 & 10.9 & 2.2 & 1.8 & 0.0 & 0.0 & 0.0 & 0.0 & 0.0 & \\
\hline$\nu b j j$ & 4077.8 & 985.1 & 13.3 & 0.4 & 0.1 & 0.1 & 0.0 & 0.0 & 0.0 & 0.0 & 0.0 & \\
\hline$\nu t b$ & 84395.2 & 51227.5 & 3848.3 & 405.4 & 73.3 & 70.6 & 0.7 & 0.7 & 0.0 & 0.0 & 0.0 & \\
\hline$\nu j j j$ & 3870920.0 & 718974.7 & 1675.5 & 0.0 & 0.0 & 0.0 & 0.0 & 0.0 & 0.0 & 0.0 & 0.0 & \\
\hline $\bar{e} e 2,70.59$ & 512.1 & 219.2 & 206.2 & 28.4 & $\bar{~} 13.6$ & 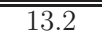 & 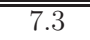 & 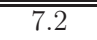 & 3.8 & ב3.6 & 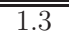 & $0.33(1.0)$ \\
\hline$e b b j$ & 2688390.0 & 176102.0 & 135388.5 & 15530.5 & 3505.9 & 3222.5 & 2357.2 & 2222.9 & 402.8 & 223.8 & 14.9 & \multirow{8}{*}{$\mathrm{B}=16.0$} \\
\hline$e b j j$ & 330834.0 & 31317.5 & 23381.6 & 575.6 & 142.3 & 100.9 & 64.5 & 64.5 & 3.3 & 0.8 & 0.8 & \\
\hline$e t \bar{t}$ & 1425.5 & 1313.8 & 1136.9 & 131.8 & 20.9 & 20.7 & 8.2 & 8.1 & 0.5 & 0.5 & 0.3 & \\
\hline$e j j j$ & 37224100.0 & 4943049.0 & 3940097.5 & 8862.8 & 1611.4 & 1128.0 & 1128.0 & 1128.0 & 161.1 & 0.0 & 0.0 & \\
\hline$\nu b b j$ & 21385.4 & 4040.5 & 112.0 & 10.9 & 1.7 & 1.3 & 0.0 & 0.0 & 0.0 & 0.0 & 0.0 & \\
\hline$\nu b j j$ & 4077.8 & 985.1 & 13.3 & 0.4 & 0.1 & 0.1 & 0.0 & 0.0 & 0.0 & 0.0 & 0.0 & \\
\hline$\nu t b$ & 84395.2 & 51227.5 & 3848.3 & 405.4 & 74.7 & 71.9 & 2.1 & 2.1 & 0.0 & 0.0 & 0.0 & \\
\hline$\nu j j j$ & 3870920.0 & 718974.7 & 1675.5 & 0.0 & 0.0 & 0.0 & 0.0 & 0.0 & 0.0 & 0.0 & 0.0 & \\
\hline e3,75.29 & 685.0 & 310.1 & 291.6 & $\begin{array}{l}42.0 \\
\end{array}$ & 18.2 & $\begin{array}{l}17.9 \\
\end{array}$ & 10.0 & 9.8 & 4.9 & 4.7 & 1.5 & $0.38(1.2)$ \\
\hline$e b b j$ & 2688390.0 & 176102.0 & 135388.5 & 15530.5 & 3147.9 & 2894.3 & 2103.6 & 1999.1 & 373.0 & 238.7 & 14.9 & \multirow{8}{*}{$\mathrm{B}=16.0$} \\
\hline$e b j j$ & 330834.0 & 31317.5 & 23381.6 & 575.6 & 134.0 & 97.6 & 63.7 & 63.7 & 2.5 & 0.8 & 0.8 & \\
\hline$e t \bar{t}$ & 1425.5 & 1313.8 & 1136.9 & 131.8 & 21.4 & 21.1 & 8.4 & 8.3 & 0.6 & 0.5 & 0.3 & \\
\hline$e j j j$ & 37224100.0 & 4943049.0 & 3940097.5 & 8862.8 & 1611.4 & 1128.0 & 966.9 & 966.9 & 161.1 & 0.0 & 0.0 & \\
\hline$\nu b b j$ & 21385.4 & 4040.5 & 112.0 & 10.9 & 1.4 & 1.0 & 0.0 & 0.0 & 0.0 & 0.0 & 0.0 & \\
\hline$\nu b j j$ & 4077.8 & 985.1 & 13.3 & 0.4 & 0.1 & 0.1 & 0.0 & 0.0 & 0.0 & 0.0 & 0.0 & \\
\hline$\nu t b$ & 84395.2 & 51227.5 & 3848.3 & 405.4 & 78.2 & 75.4 & 2.1 & 2.1 & 0.0 & 0.0 & 0.0 & \\
\hline$\nu j j j$ & 3870920.0 & 718974.7 & 1675.5 & 0.0 & 0.0 & 0.0 & 0.0 & 0.0 & 0.0 & 0.0 & 0.0 & \\
\hline$e 4,82.24$ & 4433.8 & 210.9 & "198.6 & 28.0 & 10.8 & "10.6 & 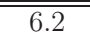 & "6.0 & 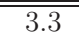 & 3.1 & 1.1 & $=0.28(0.9)$ \\
\hline$e b b j$ & 2688390.0 & 176102.0 & 135388.5 & 15530.5 & 2745.1 & 2566.0 & 1879.8 & 1849.9 & 432.6 & 283.5 & 14.9 & \multirow{8}{*}{$\mathrm{B}=15.3$} \\
\hline$e b j j$ & 330834.0 & 31317.5 & 23381.6 & 575.6 & 130.7 & 93.5 & 60.4 & 60.4 & 1.7 & 0.0 & 0.0 & \\
\hline$e t \bar{t}$ & 1425.5 & 1313.8 & 1136.9 & 131.8 & 21.3 & 21.1 & 8.3 & 8.2 & 0.6 & 0.6 & 0.4 & \\
\hline$e j j j$ & 37224100.0 & 4943049.0 & 3940097.5 & 8862.8 & 1450.3 & 1128.0 & 966.9 & 966.9 & 322.3 & 161.1 & 0.0 & \\
\hline$\nu b b j$ & 21385.4 & 4040.5 & 112.0 & 10.9 & 1.2 & 0.7 & 0.0 & 0.0 & 0.0 & 0.0 & 0.0 & \\
\hline$\nu b j j$ & 4077.8 & 985.1 & 13.3 & 0.4 & 0.1 & 0.1 & 0.0 & 0.0 & 0.0 & 0.0 & 0.0 & \\
\hline$\nu t b$ & 84395.2 & 51227.5 & 3848.3 & 405.4 & 78.9 & 78.2 & 2.1 & 2.1 & 0.0 & 0.0 & 0.0 & \\
\hline$\nu j j j$ & 3870920.0 & 718974.7 & 1675.5 & 0.0 & 0.0 & 0.0 & 0.0 & 0.0 & 0.0 & 0.0 & 0.0 & \\
\hline e $e 5,88.07$ & 383.2 & 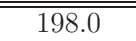 & 186.2 & 27.2 & 9.5 & 9.3 & 5.2 & 6.1 & 2.6 & 2.6 & 0.83 & $0.12(0.4)$ \\
\hline$e b b j$ & 2688390.0 & 176102.0 & 135388.5 & 15530.5 & 2700.3 & 2506.4 & 1894.7 & 1820.1 & 343.1 & 253.6 & 44.8 & \multirow{8}{*}{$\mathrm{B}=45.2$} \\
\hline$e b j j$ & 330834.0 & 31317.5 & 23381.6 & 575.6 & 112.5 & 80.2 & 49.6 & 48.8 & 3.3 & 2.5 & 0.0 & \\
\hline$e t \bar{t}$ & 1425.5 & 1313.8 & 1136.9 & 131.8 & 21.4 & 21.2 & 8.3 & 8.2 & 0.7 & 0.7 & 0.4 & \\
\hline$e j j j$ & 37224100.0 & 4943049.0 & 3940097.5 & 8862.8 & 966.9 & 966.9 & 805.7 & 805.7 & 322.3 & 161.1 & 0.0 & \\
\hline$\nu b b j$ & 21385.4 & 4040.5 & 112.0 & 10.9 & 1.1 & 0.8 & 0.0 & 0.0 & 0.0 & 0.0 & 0.0 & \\
\hline$\nu b j j$ & 4077.8 & 985.1 & 13.3 & 0.4 & 0.1 & 0.1 & 0.0 & 0.0 & 0.0 & 0.0 & 0.0 & \\
\hline$\nu t b$ & 84395.2 & 51227.5 & 3848.3 & 405.4 & 86.5 & 85.8 & 1.4 & 1.4 & 0.0 & 0.0 & 0.0 & \\
\hline$\nu j j j$ & 3870920.0 & 718974.7 & 1675.5 & 0.0 & 0.0 & 0.0 & 0.0 & 0.0 & 0.0 & 0.0 & 0.0 & \\
\hline
\end{tabular}

Table 4: The number of signal and backgrounds events $e+3$-jet channel after cumulative set of selections at the LHeC collider with $100 \mathrm{fb}^{-1}$ integrated luminosity for selective Benchmark points consistent with all the Phenomenological, Sparticle masses, Relic density of cold-dark matter, direct and indirect dark matter searches and most up-to-dated Higgs boson data. We simulated each benchmark signal with $200 \mathrm{~K}$ and each background with $400 \mathrm{~K}$ Monte Carlo simulated events. In the second column "RawEvt" stands for the number of events with only the generator-level cuts (Eq11) imposed for the signal as well as for backgrounds. For signal benchmarks, the proper branching factor, $h_{1} \rightarrow b \bar{b}$ has been multiplied with the total cross section and for backgrounds with $W$-boson and $t$-quark we assume a free decay. In the final column we list the $\operatorname{significances}(\mathcal{S})$ defined as $\mathcal{S}=S / \sqrt{B}$, where $S(B)$ stands for signal (background) events for $100 \mathrm{fb}^{-1}$ of data after all cuts mentioned in the "j" column are implemented. The Significances for $1000 \mathrm{fb}^{-1}$ are shown in the parenthesis. 


\begin{tabular}{||c||c|c|c|c||c|c|c|c||}
\hline \hline & & & & & & \\
$\mathrm{BP}, m_{h}$ & $\eta_{l}, \Delta \eta_{j l}, m_{\phi j}, H_{T}, \vec{H}_{T}$ & $\mathrm{~S}$ & $\mathrm{~B}$ & $\mathcal{S}$ & $\eta_{l}, \Delta \eta_{j l}, m_{\phi j}, H_{T}, \vec{H}_{T}$ & $\mathrm{~S}$ & $\mathrm{~B}$ & $\mathcal{S}$ \\
\hline$e 1,63.59$ & $(1.0,-1.0),(0.0,-4.3), 180,130,60$ & 4.9 & 162.3 & $0.38(1.2)$ & $(1.6,-2.5),(0.3,-6.0), 100,140,30$ & 12.8 & 412.7 & $0.63(1.99)$ \\
$e 2,70.59$ & $(1.0,-1.0),(0.0,-3.0), 180,140,60$ & 2.7 & 1.3 & $2.36(7.5)$ & $(1.1,-2.5),(0.2,-5.7), 90,90,30$ & 10.1 & 1295.3 & $0.28(0.89)$ \\
$e 3,75.29$ & $(1.0,-2.5),(0.4,-3.4), 180,140,60$ & 3.1 & 1.5 & $2.53(8.0)$ & $(1.0,-2.1),(0.4,-6.0), 120,110,30$ & 11.6 & 565.2 & $0.49(1.54)$ \\
$e 4,82.24$ & $(1.0,-1.4),(0.0,-3.4), 180,140,60$ & 1.6 & 0.6 & $2.09(6.6)$ & $(1.0,-2.1),(0.1,-3.4), 110,140,30$ & 4.1 & 154.1 & $0.32(1.0)$ \\
$e 5,88.07$ & $(1.0,-1.8),(0.0,-3.0), 180,140,60$ & 1.3 & 2.4 & $0.85(2.7)$ & $(1.3,-2.1),(0.1,-5.9), 150,140,30$ & 4.8 & 340.0 & $0.26(0.82)$ \\
\hline \hline
\end{tabular}

Table 5: The optimization of the signal channel with different sets of kinematical selection cuts, e.g., $\eta_{l}, \Delta \eta_{j l}, m_{\phi j}, H_{T}$, magnitude of vector $\vec{H}_{T}$ (see text for details) with the best significance obtained for $100 \mathrm{fb}^{-1}$. In the right-sided columns we required strictly that the number of signal events must be greater than 10 or at least approximately 5 for the low luminosity options. The significances in the parenthesis are for $1000 \mathrm{fb}^{-1}$. 


\begin{tabular}{|c|c|c|c|c|c|c|c|c|c|c|c|}
\hline Proc, $m_{h}$ & RawEvt & A & B & $\mathrm{C}$ & $\mathrm{D}$ & $\mathrm{E}$ & $\mathrm{F}$ & G & $\mathrm{H}$ & I & $\mathcal{S}$ \\
\hline $\bar{\nu} \nu-1,65.93$ & 4114.1 & 1540.7 & 1475.6 & 200.6 & "112.5 & "111.2 & 102.0 & 499.8 & 477.8 & 26.0 & (3.34(10.6) \\
\hline$\nu b b j$ & 21385.4 & 4040.5 & 3928.5 & 244.7 & 43.0 & 33.1 & 32.1 & 5.3 & 5.2 & 4.1 & \multirow{8}{*}{$\mathrm{B}=60.5$} \\
\hline$\nu b j j$ & 4077.8 & 985.1 & 971.8 & 16.4 & 4.6 & 3.0 & 2.8 & 0.1 & 0.1 & 0.0 & \\
\hline$\nu t b$ & 84395.2 & 51227.5 & 47379.2 & 3671.2 & 709.1 & 661.3 & 598.4 & 27.7 & 26.3 & 12.5 & \\
\hline$\nu j j j$ & 3870920.0 & 718974.7 & 717299.2 & 722.2 & 173.3 & 144.4 & 144.4 & 28.9 & 28.9 & 28.9 & \\
\hline$e b b j$ & 2688390.0 & 176148.3 & 40728.9 & 5302.1 & 1389.0 & 1254.6 & 507.8 & 149.4 & 104.5 & 14.9 & \\
\hline$e b j j$ & 330834.0 & 31317.5 & 7935.9 & 214.2 & 58.7 & 34.7 & 11.6 & 0.8 & 0.8 & 0.0 & \\
\hline$e t \bar{t}$ & 1425.5 & 1313.8 & 176.9 & 21.7 & 3.0 & 3.0 & 2.5 & 0.1 & 0.1 & 0.1 & \\
\hline$e j j j$ & 37224100.0 & 4943049.0 & 1002951.5 & 3706.3 & 644.6 & 644.6 & 0.0 & 0.0 & 0.0 & 0.0 & \\
\hline$\nu-2,71.32$ & 2323.5 & $\begin{array}{l}917.2 \\
\end{array}$ & 879.1 & 123.5 & "61.4 & 60.9 & 55.8 & 27.3 & 26.1 & 14.2 & $1.84(5.8)$ \\
\hline$\nu b b j$ & 21385.4 & 4040.5 & 3928.5 & 244.7 & 35.9 & 28.2 & 27.2 & 4.5 & 4.5 & 3.5 & \multirow{8}{*}{$\mathrm{B}=59.7$} \\
\hline$\nu b j j$ & 4077.8 & 985.1 & 971.8 & 16.4 & 4.3 & 2.9 & 2.6 & 0.1 & 0.1 & 0.0 & \\
\hline$\nu t b$ & 84395.2 & 51227.5 & 47379.2 & 3671.2 & 727.0 & 681.4 & 626.0 & 31.8 & 30.4 & 17.3 & \\
\hline$\nu j j j$ & 3870920.0 & 718974.7 & 717299.2 & 722.2 & 202.2 & 173.3 & 173.3 & 28.9 & 28.9 & 28.9 & \\
\hline$e b b j$ & 2688390.0 & 176148.3 & 40728.9 & 5302.1 & 1344.2 & 1209.8 & 433.1 & 89.6 & 59.7 & 0.0 & \\
\hline$e b j \underline{j}$ & 330834.0 & 31317.5 & 7935.9 & 214.2 & 59.5 & 36.4 & 15.7 & 0.8 & 0.8 & 0.0 & \\
\hline$e t \bar{t}$ & 1425.5 & 1313.8 & 176.9 & 21.7 & 3.3 & 3.3 & 2.8 & 0.1 & 0.1 & 0.0 & \\
\hline$e j j j$ & 37224100.0 & 4943049.0 & 1002951.5 & 3706.3 & 483.4 & 322.3 & 0.0 & 0.0 & 0.0 & 0.0 & \\
\hline $\bar{\nu} \nu-3,83.77$ & 1830.6 & 845.3 & 809.8 & "116.0 & 44.0 & 43.4 & 40.0 & 21.2 & 20.7 & 11.1 & $1.54(4.9)$ \\
\hline$\nu b b j$ & 21385.4 & 4040.5 & 3928.5 & 244.7 & 24.2 & 20.0 & 19.5 & 3.8 & 3.8 & 3.0 & \multirow{8}{*}{$\mathrm{B}=52.1$} \\
\hline$\nu b j j$ & 4077.8 & 985.1 & 971.8 & 16.4 & 3.2 & 2.2 & 2.0 & 0.1 & 0.1 & 0.1 & \\
\hline$\nu t b$ & 84395.2 & 51227.5 & 47379.2 & 3671.2 & 736.0 & 695.9 & 643.3 & 36.0 & 35.3 & 20.1 & \\
\hline$\nu j j j$ & 3870920.0 & 718974.7 & 717299.2 & 722.2 & 202.2 & 173.3 & 173.3 & 28.9 & 28.9 & 28.9 & \\
\hline$e b b j$ & 2688390.0 & 176148.3 & 40728.9 & 5302.1 & 1015.6 & 896.1 & 388.3 & 104.5 & 74.7 & 0.0 & \\
\hline$e b j j$ & 330834.0 & 31317.5 & 7935.9 & 214.2 & 38.9 & 25.6 & 13.2 & 0.0 & 0.0 & 0.0 & \\
\hline$e t \bar{t}$ & 1425.5 & 1313.8 & 176.9 & 21.7 & 3.6 & 3.6 & 2.9 & 0.2 & 0.2 & 0.0 & \\
\hline$e j j j$ & 37224100.0 & 4943049.0 & 1002951.5 & 3706.3 & 322.3 & 0.0 & 0.0 & 0.0 & 0.0 & 0.0 & \\
\hline$\nu-4,88.07$ & 2018.0 & 975.7 & 9932.1 & "133.8 & 477.0 & 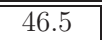 & 42.8 & 23.9 & 23.5 & $\overline{12.6}$ & $1.75(5.5)$ \\
\hline$\nu b b j$ & 21385.4 & 4040.5 & 3928.5 & 244.7 & 22.0 & 18.3 & 18.0 & 3.6 & 3.6 & 2.8 & \multirow{8}{*}{$\mathrm{B}=52.0$} \\
\hline$\nu b j j$ & 4077.8 & 985.1 & 971.8 & 16.4 & 3.0 & 2.0 & 1.9 & 0.1 & 0.1 & 0.1 & \\
\hline$\nu t b$ & 84395.2 & 51227.5 & 47379.2 & 3671.2 & 720.1 & 680.0 & 624.7 & 34.6 & 33.2 & 20.1 & \\
\hline$\nu j j j$ & 3870920.0 & 718974.7 & 717299.2 & 722.2 & 144.4 & 115.5 & 115.5 & 28.9 & 28.9 & 28.9 & \\
\hline$e b b j$ & 2688390.0 & 176148.3 & 40728.9 & 5302.1 & 970.8 & 866.3 & 403.3 & 104.5 & 74.7 & 0.0 & \\
\hline$e b j j$ & 330834.0 & 31317.5 & 7935.9 & 214.2 & 41.4 & 28.1 & 16.5 & 0.8 & 0.8 & 0.0 & \\
\hline$e t \bar{t}$ & 1425.5 & 1313.8 & 176.9 & 21.7 & 3.8 & 3.8 & 3.0 & 0.2 & 0.2 & 0.1 & \\
\hline$e j j j$ & 37224100.0 & 4943049.0 & 1002951.5 & 3706.3 & 161.1 & 0.0 & 0.0 & 0.0 & 0.0 & 0.0 & \\
\hline$\nu-5,100.47$ & 1125.0 & $6 \quad 613.9$ & 585.0 & 88.2 & 23.5 & 23.3 & 21.5 & $\bar{~} 12.0$ & 11.9 & 6.1 & $1.41(4.5)$ \\
\hline$\nu b b j$ & 21385.4 & 4040.5 & 3928.5 & 244.7 & 15.0 & 11.8 & 11.6 & 2.4 & 2.4 & 1.9 & \multirow{8}{*}{$\mathrm{B}=18.7$} \\
\hline$\nu b j j$ & 4077.8 & 985.1 & 971.8 & 16.4 & 1.9 & 1.3 & 1.3 & 0.1 & 0.1 & 0.1 & \\
\hline$\nu t b$ & 84395.2 & 51227.5 & 47379.2 & 3671.2 & 644.0 & 615.0 & 562.4 & 39.4 & 38.7 & 16.6 & \\
\hline$\nu j j j$ & 3870920.0 & 718974.7 & 717299.2 & 722.2 & 115.5 & 86.7 & 86.7 & 28.9 & 28.9 & 0.0 & \\
\hline$e b b j$ & 2688390.0 & 176148.3 & 40728.9 & 5302.1 & 806.5 & 672.1 & 313.6 & 59.7 & 29.9 & 0.0 & \\
\hline$e b j j$ & 330834.0 & 31317.5 & 7935.9 & 214.2 & 25.6 & 17.4 & 11.6 & 2.5 & 2.5 & 0.0 & \\
\hline$e t \bar{t}$ & 1425.5 & 1313.8 & 176.9 & 21.7 & 4.0 & 3.9 & 3.0 & 0.2 & 0.2 & 0.1 & \\
\hline$e j j j$ & 37224100.0 & 4943049.0 & 1002951.5 & 3706.3 & 483.4 & 322.3 & 0.0 & 0.0 & 0.0 & 0.0 & \\
\hline
\end{tabular}

Table 6: Same as of previous table but for $\mathbb{E}_{T}+3$-jet channel. In the final column we mention the significances $(\mathcal{S})$ defined as $\mathcal{S}=S / \sqrt{B}$, where $S(B)$ for signal (background) events for 100 $\mathrm{fb}^{-1}$ of data after all cuts mentioned in the "I" column are implemented. The significances for $1000 \mathrm{fb}^{-1}$ are shown in the parenthesis. 


\begin{tabular}{||c||c|c|c|c||c|c|c|c||}
\hline \hline $\mathrm{BP}, m_{h}$ & $E_{T}, m_{\phi j}, H_{T}, \vec{H}_{T}$ & $\mathrm{~S}$ & $\mathrm{~B}$ & $\mathcal{S}$ & $\mathscr{E}_{T}, m_{\phi j}, H_{T}, \vec{H}_{T}$ & $\mathrm{~S}$ & $\mathrm{~B}$ & $\mathcal{S}$ \\
\hline$\nu 1,65.93$ & $35,180,70,30$ & 44.9 & 90.7 & $4.7(14.9)$ & $10,170,70,60$ & 28.6 & 63.9 & $3.57(11.4)$ \\
$\nu 2,71.32$ & $35,180,70,30$ & 25.3 & 83.2 & $2.8(8.9)$ & $40,170,70,50$ & 19.6 & 75.7 & $2.3(7.2)$ \\
$\nu 3,83.77$ & $30,180,70,30$ & 20.1 & 97.4 & $2.0(6.5)$ & $30,180,90,30$ & 19.9 & 96.7 & $2.0(6.5)$ \\
$\nu 4,88.07$ & $30,180,90,30$ & 23.5 & 97.6 & $2.4(7.6)$ & $35,180,120,30$ & 19.6 & 92.3 & $2.0(6.5)$ \\
$\nu 5,100.47$ & $30,180,100,30$ & 12.3 & 105.8 & $1.2(3.8)$ & $25,180,100,50$ & 7.9 & 45.0 & $1.2(3.7)$ \\
\hline \hline
\end{tabular}

Table 7: The optimization of the signal channel with different sets of selection cuts, e.g., $\mathbb{E}_{T}$, $m_{\phi j}, H_{T}$, the magnitude of $\vec{H}_{T}$ (see text for details) with the best significance obtained for 100 $\mathrm{fb}^{-1}$. The significances in the parenthesis are for $1000 \mathrm{fb}^{-1}$. 